\title{
Gradient conjugate priors and multi-layer neural networks
}

\author{
Pavel Gurevich* Hannes Stuke
}

March 27, 2019

\begin{abstract}
The paper deals with learning probability distributions of observed data by artificial neural networks. We suggest a so-called gradient conjugate prior (GCP) update appropriate for neural networks, which is a modification of the classical Bayesian update for conjugate priors. We establish a connection between the gradient conjugate prior update and the maximization of the log-likelihood of the predictive distribution. Unlike for the Bayesian neural networks, we use deterministic weights of neural networks, but rather assume that the ground truth distribution is normal with unknown mean and variance and learn by the neural networks the parameters of a prior (normal-gamma distribution) for these unknown mean and variance. The update of the parameters is done, using the gradient that, at each step, directs towards minimizing the Kullback-Leibler divergence from the prior to the posterior distribution (both being normal-gamma). We obtain a corresponding dynamical system for the prior's parameters and analyze its properties. In particular, we study the limiting behavior of all the prior's parameters and show how it differs from the case of the classical full Bayesian update. The results are validated on synthetic and real world data sets.
\end{abstract}

Keywords. Conjugate priors, Kullback-Leibler divergence, latent variables, Student's t-distribution, deep neural networks, regression, uncertainty quantification, asymptotics, outliers

\section{Contents}

1 Introduction

*Free University of Berlin, Arnimallee 3, 14195 Berlin, Germany; RUDN University, Miklukho-Maklaya 6, 117198 Moscow, Russia; email: gurevich@math.fu-berlin.de

${ }^{\dagger}$ Free University of Berlin, Arnimallee 7, 14195 Berlin, Germany; email: h.stuke@fu-berlin.de

${ }^{\ddagger}$ Equal contribution. 
2 Motivation 6

2.1 Estimating normal distributions with unknown mean and precision . . . . 6 6

2.2 Conjugate prior update . . . . . . . . . . . . . . . 7

2.3 Maximization of the predictive distribution and the GCP update . . . . . 8

2.4 Practical approaches . . . . . . . . . . . . . . . . . . 10 10

3 Dynamics of $m, \alpha, \beta, \nu$

3.1 Dynamical system for $m, \alpha, \beta, \nu \ldots \ldots \ldots \ldots \ldots \ldots \ldots \ldots \ldots$

3.2 Estimation of the mean $m \ldots \ldots \ldots \ldots$. . . . . . . . . . . . . . .

3.3 Estimation of the variance. The unbounded absorbing set . . . . . . . . 13

3.3.1 The functions $A(\alpha)$ and $\sigma_{\varkappa}(\alpha) \ldots \ldots \ldots \ldots \ldots$

3.3 .2 Estimation of the variance . . . . . . . . . . . . . 15

3.4 Dynamics of $\alpha, \beta, \nu$. Proof of Theorem $3.2 \ldots \ldots \ldots \ldots$

4 Dynamics of $m, \beta, \nu$ for a fixed $\alpha \quad 19$

4.1 Estimation of the variance. The curves of equilibria . . . . . . . . . 19

5 Role of a fixed $\alpha \quad 2$

5.1 Sensitivity to outliers . . . . . . . . . . . . . . . . . 22

5.2 Learning speed in clean and noisy regions . . . . . . . . . . . . 22

5.2 .1 Observations . . . . . . . . . . . . . . . . . 22

5.2 .2 Justification of the observations . . . . . . . . . . . . . 24

6 GCP neural networks: experiments 26

6.1 Methods . . . . . . . . . . . . . . . . . . . . [26

6.2 Synthetic data set: aleatoric vs. epistemic uncertainty . . . . . . . . 26

6.3 Synthetic data set: robustness to outliers in the training set . . . . . . . . 27

6.4 Real world data sets . . . . . . . . . . . . . . . . . . . 28 28

6.5 Real world data sets: outliers in the training data sets . . . . . . . . . . . 30

7 Conclusion 31

A Properties of the function $A(\alpha)$ : proof of Lemma 3.1

B Proof of Lemma 3.2

C Proof of Lemma 3.3

D Proof of Lemma 3.4

E Hyperparameters 


\section{Introduction}

Reconstructing probability distributions of observed data by artificial neural networks is one of the most essential parts of machine learning and artificial intelligence [3, 33]. Learning probability distributions not only allows one to predict the behavior of a system under consideration, but to also quantify the uncertainty with which the predictions are done. Under the assumption that the data are normally distributed, the most well studied way of reconstructing probability distributions is the Bayesian learning of neural networks [31]. One treats the weights of the network as normally distributed random variables, prescribes their prior distribution, and then finds the posterior distribution conditioned on the data. The main difficulty is that neither the posterior, nor the resulting predictive distributions are given in a closed form. As a result, different approximation methods have been developed [4, 7, 8, $13,15,17,21,25,26,28,29,36,46$. However, many of them have certain drawbacks related to the lack of scalability in data size or the neural network complexity, and are still a field of ongoing research. Furthermore, Bayesian neural networks often assume homoscedastic variance in the likelihood (i.e., same for all samples) and rather learn uncertainty due to lack of data (epistemic uncertainty). Among other methods for uncertainty quantification, there are the delta method [16, 45, 47], the mean-variance estimate [37], and deep ensemble methods [23, 24]. A combination of the Bayesian approach (using the dropout variational inference) with the mean-variance estimate was used in [19], thus allowing for a simultaneous estimation of epistemic and aleatoric (due to noise in data) uncertainty. A new method based on minimizing a joint loss for a regression network and another network quantifying uncertainty was recently proposed in [1]. We refer to [20, 42] and the recent works [7, 11, 23, 24, 34 for a comprehensive comparison of the above methods and further references to research on the Bayesian learning of neural networks.

We study an alternative approach to reconstructing the ground truth probability distribution based on what we call a gradient conjugate prior $(G C P)$ update. We are interested in learning conditional probability distributions $q(y \mid x)$ of $\operatorname{target}^{1} \mathbf{y} \in \mathbb{R}$ corresponding to data samples $x \in \mathbb{R}^{m}$, using artificial neural networks (supervised learning). For brevity, we will often omit the dependence of distributions on $x$. Thus, assuming that the ground truth distribution $q(y)$ of a random variable $\mathbf{y}$ (corresponding to observed data) is Gaussian with unknown mean and precision, we let neural networks learn the four parameters of the normal-gamma distribution that serves as a prior for the mean and variance of $\mathbf{y}$. We emphasize that, unlike for Bayesian neural networks, the weights of the neural networks are deterministic in our approach. Given a parametrized prior, one has the predictive distribution in the form of a (non-standardized) Student's t-distribution $p_{\text {pred }}(y)$, whose parameters are explicitly determined by the outputs of the neural networks. For further details, we refer to Sec. 2, which includes a graphical model visualization in Fig. 2.1 and a comparison with Bayesian neural networks in Table 2.1.

Given an observation $\left(x_{n}, y_{n}\right)(n=1, \ldots, N)$, the classical Bayesian update yields the

\footnotetext{
${ }^{1}$ Throughout this paper, we denote random variables by bold letters and the arguments of their probability distributions by the corresponding non-bold letters.
} 
posterior distribution for the mean and variance of $\mathbf{y}_{n}$. This posterior appears normal-gamma as well [3]. However, one cannot update its parameters directly because they are represented by the outputs of the neural networks. Instead, one has to update the weights of the neural networks. We suggest to make a gradient descent step in the direction of minimization of the Kullback-Leibler (KL) divergence from the prior to the posterior (see the details in Sec. 2 ). This is the step that we call the GCP update. After updating the weights, one takes the next observation $\left(x_{n+1}, y_{n+1}\right)$ and repeats the above update procedure. One cycles over the whole training data set until convergence of the log-likelihood of predictive distribution

$$
\frac{1}{N} \sum_{n=1}^{N} \ln p_{\text {pred }}\left(y_{n} \mid x_{n}\right) .
$$

In the paper, we provide a detailed analysis of the dynamics given by the GCP update. Intuitively, one might think that the GCP update, after convergence, yields the same result as the classical CP update. Surprisingly, this is not the case: the parametrized normal-gamma distribution does not converge to the Bayesian posterior (see Remark 3.4). Nevertheless, the predictive distribution does converge to the ground truth Gaussian distribution $q(y)$. This is explained by the observation, which we prove in Sec. 2.3. the GCP update is actually equivalent to maximizing by gradient ascent the log-likelihood (1.1) of the predictive distribution. As the number of observations tends to infinity the GCP update becomes also equivalent to minimizing by gradient descent the $K L$ divergence from the predictive distribution $p_{\text {pred }}(y)$ to the ground truth distribution $q(y)$. We show that these equivalences hold in general, even if the prior is not conjugate to the likelihood function. Thus, we see that the GCP method estimates aleatoric uncertainty.

We emphasize that, although in our approach the approximating distribution gets parametrized (as the predictive distribution in the mean-variance approach [37] or the approximating latent variables distribution in variational autoencoders $[22]$ ), the way we parametrize and optimize and the way we interpret the result is different, as shown in Fig. 2.1 and summarized in Table 2.1.

Now let us come back to our original assumption that $q(y)$ is a normal distribution and $p_{\text {pred }}(y)$ is a Student's t-distribution. The latter appears to be overparametrized (by four parameters instead of three). We keep it overparametrized in order to compare the dynamics of the parameters under the classical CP update and under the GCP update. Reformulation of our results for Student's t-distribution parameterized in the standard way by three parameters will be straightforward. There is a vast literature on the estimation of parameters of Student's t-distribution, see, e.g., the overview [35] and the references therein. Note that, in the context of neural networks, different samples correspond to different inputs of the network, and hence they belong to different Student's t-distributions with different unknown parameters. Thus, the maximization of the likelihood of Student's t-distribution with respect to the weights of the networks is one of the most common methods. In [44], the possibility of utilizing evolutionary algorithms for maximizing the likelihood was explored experimentally. Another natural way is to use the gradient ascent with respect to the weights of the network. As we said, the latter is equivalent to the usage of the GCP update. In the 
paper, we obtain a dynamical system for the prior's parameters that approximates the GCP update (as well as the gradient ascent for maximization of Student's t-distribution). We study the dynamics of the prior's parameters in detail, in particular analyzing their convergence properties. Our approach is illustrated with synthetic data and validated on various realworld data sets in comparison with other methods for learning probability distributions based on neural networks. To our best knowledge, neither the dynamical systems analysis of the GCP (or gradient ascent for maximizing the likelihood of Student's t-distribution), nor a thorough comparison of the GCP with other methods has been carried out before.

As an interesting and useful consequence of our analysis, we will see how the GCP interacts with the output outliers in the training set (a small percentage of observations that do not come from the assumed normal distribution $q(y)$ ). The outliers prevent one of the prior's parameters $\left(\alpha\right.$, which is related to the number of degrees of freedom of $\left.p_{\text {pred }}(y)\right)$ from going to infinity. On one hand, this is known [30,40] to allow for a better estimate of the mean and variance of $q(y)$, compared with directly using the maximization of the likelihood of a normal distribution. However, on the other hand, this still leads to overestimation of the variance of $q(y)$. To deal with this issue, we obtain an explicit formula (see (2.18) ) that allows one to correct the estimate of the variance and recover the ground truth variance of $q(y)$. To our knowledge, such a correction formula was not derived in the literature before.

The paper is organized as follows. In Sec. 2, we provide a detailed motivation for the GCP update, explain how we approximate the parameters of the prior distribution by neural networks, establish the relation between the GCP update and the predictive distribution, and formulate the method of learning the ground truth distribution from the practical point of view. Section 3 is the mathematical core of this paper. We derive a dynamical system for the prior's parameters, induced by the GCP update, and analyze it in detail. In particular, we obtain an asymptotics for the growth rate of $\alpha$ and find the limits of the other parameters of the prior. In Sec. 4, we study the dynamics for a fixed $\alpha$. We find the limiting values for the rest of the parameters and show how one can recover the variance of the ground truth normal distribution $q(y)$. In Sec. 5, we clarify the role of a fixed $\alpha$. Namely, we compare the sensitivity to output outliers of the GCP update with that in minimizing the standard squared error loss or maximizing the log-likelihood of a normal distribution. Furthermore, we show how $\alpha$ controls the learning speed in clean and noisy regions. In Sec. 6, we illustrate the fit of neural networks for synthetic and various real-world data sets. Section 7 contains a conclusion and an outline of possible directions of further research. Appendices $\mathrm{A}, \mathrm{D}$ contain the proofs of auxiliary lemmas from Sec. 3. In Appendix E, we present the values of hyperparameters of different methods that are compared in Sec. 6 . 


\section{Motivation}

\subsection{Estimating normal distributions with unknown mean and pre- cision}

Assume one wants to estimate unknown mean and precision (the inverse of the variance) of normally distributed data $\mathbf{y} \mid x$. We remind that $\mathbf{y}$ is conditioned on $x \in \mathbb{R}^{m}$, but we often omit this dependence in our notation. We will analyze scalar $\mathbf{y}$ and refer to Sec. 7 for a discussion of multivariate data. One standard approach for estimating the mean and precision is based on conjugate priors. One assumes that the mean and precision are random variables, $\boldsymbol{\mu}$ and $\boldsymbol{\tau}$ respectively, with a joint prior given by the normal-gamma distribution

$$
p(\mu, \tau \mid m, \nu, \alpha, \beta)=\frac{\beta^{\alpha} \nu^{1 / 2}}{\Gamma(\alpha)(2 \pi)^{1 / 2}} \tau^{\alpha-1 / 2} e^{-\beta \tau} e^{-\frac{\nu \tau(\mu-m)^{2}}{2}},
$$

where $m \in \mathbb{R}, \nu>0, \alpha>1, \beta>0$.

The marginal distribution for $\boldsymbol{\mu}$ is a non-standardized Student's t-distribution with

$$
\mathbb{E}[\boldsymbol{\mu}]=m, \quad \mathbb{V}[\boldsymbol{\mu}]=\frac{\beta}{\nu(\alpha-1)} .
$$

The marginal distribution for $\boldsymbol{\tau}$ is the Gamma distribution with

$$
\mathbb{E}[\boldsymbol{\tau}]=\frac{\alpha}{\beta}, \quad \mathbb{V}[\boldsymbol{\tau}]=\frac{\mathbb{E}(\tau)}{\beta}=\frac{\alpha}{\beta^{2}} .
$$

By marginalizing $\boldsymbol{\mu}$ and $\boldsymbol{\tau}$, one obtains the predictive distribution $p_{\text {pred }}(y)=p_{\text {pred }}(y ; m, \nu, \alpha, \beta)$ for $\mathbf{y}$, which appears to be a non-standardized Student's t-distribution. Its mean and variance can be used to estimate the mean and variance of $\mathbf{y}$. The estimated mean $m_{\text {est }}$ and variance $V_{\text {est }}$ are given by

$$
m_{\mathrm{est}}:=m, \quad V_{\mathrm{est}}:=\frac{\beta(\nu+1)}{(\alpha-1) \nu} .
$$

We refer, e.g., to [3] for further details.

Our goal is to approximate the parameters $m, \alpha, \beta, \nu$ by multi-layer neural networks, i.e., to represent them as functions of inputs and weights: $m=m\left(x, w_{1}\right), \alpha=\alpha\left(x, w_{2}\right), \beta=$ $\beta\left(x, w_{3}\right), \nu=\nu\left(x, w_{4}\right), w_{j} \in \mathbb{R}^{M_{j}}$. The corresponding graphical model is shown in Fig. 2.1.

A standard way to estimate the parameters $w$ of the neural networks is to maximize the log-likelihood

$$
\frac{1}{N} \sum_{n=1}^{N} p_{\text {pred }}\left(y_{n} ; m\left(x_{n}, w_{1}\right), \alpha\left(x_{n}, w_{2}\right), \beta\left(x_{n}, w_{3}\right), \nu\left(x_{n}, w_{4}\right)\right) \rightarrow \max _{w_{1}, \ldots, w_{4}} .
$$

An alternative approach is to use the conjugacy of the prior, which allows for an explicit formula for the Kullback-Leibler (KL) divergence from the posterior to the prior. This approach is explained in Sec. 2.2, In Sec. 2.3, we show that both methods are actually equivalent. 


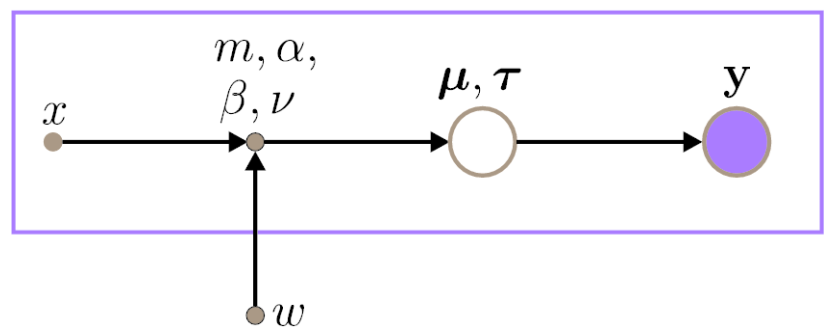

Figure 2.1: Deterministic parameters (input $x$, the prior's parameters $m, \alpha, \beta, \nu$, and the weights $w$ ) are shown by solid nodes and random variables $(\boldsymbol{\mu}, \boldsymbol{\tau}$, and $\mathbf{y})$ by circles. The shaded circle corresponds to the observed random variable $\mathbf{y}$. The box encompasses the quantities depending on $x$.

\subsection{Conjugate prior update}

Suppose one observes a new sample $y$. Then, by the Bayes theorem, the conditional distribution of $(\boldsymbol{\mu}, \boldsymbol{\tau})$ under the condition that $\mathbf{y}=y$ (the posterior distribution denoted by $\left.p_{\text {post }}(\mu, \tau)\right)$ appears to be normal-gamma as well [3], namely,

$$
p_{\text {post }}(\mu, \tau)=p\left(\mu, \tau \mid m^{\prime}, \nu^{\prime}, \alpha^{\prime}, \beta^{\prime}, y\right),
$$

where $p(\cdot)$ is defined in (2.1) and the parameters are updated as follows:

$$
m^{\prime}=\frac{\nu m+y}{\nu+1}, \quad \nu^{\prime}=\nu+1, \quad \alpha^{\prime}=\alpha+\frac{1}{2}, \quad \beta^{\prime}=\beta+\frac{\nu}{\nu+1} \frac{(y-m)^{2}}{2} .
$$

We call (2.7) the conjugate prior (CP) update.

The KL divergence from a continuous distribution $p_{\text {post }}$ to a continuous distribution $p$ is defined as follows:

$$
D_{\mathrm{KL}}\left(p_{\text {post }} \| p\right):=\int p_{\text {post }}(\mu, \tau) \ln \frac{p_{\text {post }}(\mu, \tau)}{p(\mu, \tau)} d \mu d \tau
$$

We denote by $\Psi(x):=\Gamma^{\prime}(x) / \Gamma(x)$ the digamma function, where $\Gamma(x)$ is the gamma function. Then for the above normal-gamma distributions (2.1) and (2.6) the KL divergence takes the form 41]

$$
\begin{aligned}
K(m, \nu, \alpha, \beta) & :=\frac{1}{2} \frac{\alpha^{\prime}}{\beta^{\prime}}\left(m-m^{\prime}\right)^{2} \nu+\frac{1}{2} \frac{\nu}{\nu^{\prime}}-\frac{1}{2} \ln \frac{\nu}{\nu^{\prime}}-\frac{1}{2} \\
& -\alpha \ln \frac{\beta}{\beta^{\prime}}+\ln \frac{\Gamma(\alpha)}{\Gamma\left(\alpha^{\prime}\right)}-\left(\alpha-\alpha^{\prime}\right) \Psi\left(\alpha^{\prime}\right)+\left(\beta-\beta^{\prime}\right) \frac{\alpha^{\prime}}{\beta^{\prime}} .
\end{aligned}
$$

In our case, one cannot directly apply the update in (2.7), but one must update the weights $w_{j}$ instead. The natural way to do so is to observe a sample $y$, to calculate the posterior distribution (2.6) and to change the weights $w$ in the direction of $-\nabla_{w} K$, i.e.,

$$
w_{\text {new }}:=w-\lambda \nabla_{w} K, \quad w \in\left\{w_{1}, \ldots, w_{4}\right\}
$$




\begin{tabular}{|c|c|c|}
\hline & Bayesian neural networks & GCP networks \\
\hline Data & \multicolumn{2}{|c|}{ Inputs $X=\left\{x_{1}, \ldots, x_{N}\right\}$, targets $Y=\left\{y_{1}, \ldots, y_{N}\right\}$} \\
\hline Ground truth & & Gaussian $q(y \mid x)$ \\
\hline $\begin{array}{l}\text { Captured } \\
\text { uncertainty }\end{array}$ & Epistemic, homoscedastic & Aleatoric, heteroscedastic \\
\hline Weights & Random $\boldsymbol{\theta}$ & Deterministic $w$ \\
\hline $\begin{array}{l}\text { Latent } \\
\text { variables }\end{array}$ & Weights $\boldsymbol{\theta} \sim p(\theta)$ independent of $x \in X$ & $\begin{array}{l}\text { Means and precisions }(\boldsymbol{\mu}, \boldsymbol{\tau})=\boldsymbol{\theta} \sim p(\theta \mid x, w) \\
\text { conditioned on } x \in X \text { and } w\end{array}$ \\
\hline Prior & $p(\theta)$ fixed during training & $p(\theta \mid x, w)$ evolves during training. \\
\hline Likelihood & Gaussian $\mathcal{N}\left(y \mid m(x, \theta), \tau^{-1}\right)$ with constant $\tau$ & $\begin{array}{l}\text { Gaussian } \mathcal{N}\left(y \mid \mu, \tau^{-1}\right) \text { with both } \mu \text { and } \tau \\
\text { depending on } x \in X \text { and } w \text {. }\end{array}$ \\
\hline Posterior & $p(\theta \mid X, Y)$ intractable and fixed during training & $p_{\text {post }}(\theta)$ tractable normal-Gamma and evolves during training \\
\hline Training & $\begin{array}{l}\text { Minimize the KL-divergence } D_{\mathrm{KL}}(p(\theta \mid w) \| p(\theta \mid X, Y)) \\
\text { from } p(\theta \mid w) \text { parametrized by deterministic } w \\
\text { to the intractable fixed posterior } p(\theta \mid X, Y)\end{array}$ & $\begin{array}{l}\text { Gradient descent step to minimize the reverse } \\
\text { KL-divergence } D_{\mathrm{KL}}\left(p_{\text {post }}(\theta) \| p(\theta \mid x, w)\right) \text { w.r.t. } w \text {. } \\
\text { The posterior } p_{\text {post }}(\theta) \text { is tractable and recalculated } \\
\text { after each observation }(x, y) \text { based on the evolving prior } p(\theta \mid x, w)\end{array}$ \\
\hline Result & $p(\theta \mid w)$ approximates the posterior $p(\theta \mid X, Y)$ & $\begin{array}{l}p(\theta \mid x, w) \text { does not converge to } p_{\text {post }}(\theta) \\
\text { but the predictive distribution maximizes the likelihood of data }\end{array}$ \\
\hline Predictive & $\begin{array}{l}p_{\text {pred }}(y \mid x, w)=\int \mathcal{N}\left(y \mid m(x, \theta), \tau^{-1}\right) p(\theta \mid w) d \theta \\
\text { typically evaluated by sampling }\end{array}$ & $\begin{array}{l}\text { Explicit Student's t-distribution } p_{\text {pred }}(y \mid x, w)=t_{2 \alpha}(y \mid m, \beta(\nu+1) /(\nu \alpha)) \text {, } \\
\text { where } m, \alpha, \beta, \nu \text { depend on } x \text { and } w\end{array}$ \\
\hline $\begin{array}{l}\text { Output outliers } \\
\text { in the training set }\end{array}$ & Distorted means and overestimated variances & Robust means and variances via the correction formula 2.18 \\
\hline
\end{tabular}

Table 2.1: Comparison of Bayesian neural networks with variational inference and GCP networks

where $\lambda>0$ is a learning rate. When we compute the gradient of $K$ with respect of $w$, we keep all the prime variables in (2.9) fixed and do not treat them as functions of $w$, while all the nonprime variables are treated as functions of $w$. We still use the notation $\nabla_{w} K$ in this case. We call (2.10) the gradient conjugate prior (GCP) update. In Table 2.1, we summarize our approach and highlight its difference from the Bayesian neural networks and variational inference 2 .

As we will see below, this update induces the update for $(m, \alpha, \beta, \nu)$ that is different from the classical conjugate prior update (2.7) and yields a completely different dynamics. Before we analyze this dynamics in detail, we explain why the GCP update (2.10) is equivalent to maximizing the log-likelihood (2.5) of the predictive in the general case.

\subsection{Maximization of the predictive distribution and the GCP up- date}

Suppose we want to learn a ground truth probability distribution $q(y)$ of a random variable $\mathbf{y}$ (a normal distribution in our particular case). Since the ground truth distribution is a priori unknown, we conjecture that it belongs to a family of distributions $L(y \mid \theta)$ parametrized by $\theta$ (in our case $\theta=(\mu, \tau)$ and $L(y \mid \theta)$ is a normal distribution with mean $\mu$ and precision $\tau$ ). Since $\theta$ is a priori unknown, we assume it is a random variable $\boldsymbol{\theta}$ with a prior distribution from a family $p(\theta \mid w)$ parametrized by $w$ (in our case, $p(\theta \mid w)$ is the normal-gamma distribution and $w$ are the weights of neural networks approximating $m, \alpha, \beta, \nu)$. We denote the predictive distribution by

$$
p_{\text {pred }}(y \mid w):=\int L(y \mid \theta) p(\theta \mid w) d \theta
$$

\footnotetext{
${ }^{2}$ The latent variables are usually denoted $w$ in the Bayesian neural networks framework or $\mathbf{z}$ in the variational inference framework. We use the notation $\boldsymbol{\theta}$ to make it consistent with our notation in Sec. 2.3 .
} 
(non-standardized Student's t-distribution in our case). Given an observation $\mathbf{y}=y$, the Bayes rule determines the posterior distribution of $\boldsymbol{\theta}$ :

$$
p_{\text {post }}(\theta \mid w, y):=\frac{L(y \mid \theta) p(\theta \mid w)}{p_{\text {pred }}(y \mid w)}
$$

In our case, $p_{\text {post }}(\theta \mid w, y)$ is normal-gamma again, but we emphasize that, in general, it need not be from the same family as the prior $p(\theta \mid w)$ is.

Now we compute the gradient of the KL divergence

$$
K(w, y):=D_{\mathrm{KL}}\left(p_{\text {post }} \| p\right)=\int p_{\text {post }}(\theta \mid w, y) \ln \frac{p_{\text {post }}(\theta \mid w, y)}{p(\theta \mid w)} d \theta
$$

(cf. (2.8)) with respect to $w$, assuming that $w$ in the posterior distribution is freezed, and we do not differentiate it. Denoting such a gradient by $\nabla_{w} K(w, y)$, we obtain the following lemma.

Lemma 2.1. $\nabla_{w} K(w, y)=-\nabla_{w} \ln p_{\text {pred }}(y \mid w)$.

Proof. Freezing $p_{\text {post }}(\theta \mid w, y)$ in $(2.13)$, we have

$$
\nabla_{w} K(w, y)=-\int \frac{p_{\text {post }}(\theta \mid w, y) \nabla_{w} p(\theta \mid w)}{p(\theta \mid w)} d \theta
$$

Plugging in $p_{\text {post }}(\theta \mid w, y)$ from (2.12) and using (2.11) yields

$$
\nabla_{w} K(w, y)=-\int \frac{L(y \mid \theta) \nabla_{w} p(\theta \mid w)}{p_{\text {pred }}(y \mid w)} d \theta=-\frac{\nabla_{w} p_{\text {pred }}(y \mid w)}{p_{\text {pred }}(y \mid w)}=-\nabla_{w} \ln p_{\text {pred }}(y \mid w) .
$$

Lemma 2.1 shows that the GCP update (2.10) is the gradient ascent step in the direction of maximizing the log-likelihood of the predictive distribution $p_{\text {pred }}(y \mid w)$ given a new observation $\mathbf{y}=y$. Furthermore, using Lemma 2.1, we see that given observations $y_{1}, \ldots, y_{N}$, the averaged GCP update of the parameters $w$ is given by (cf. (2.10)

$$
w_{\text {new }}:=w-\lambda \frac{1}{N} \sum_{n=1}^{N}\left[\nabla_{w} K\left(w, y_{n}\right)\right]=w+\lambda \nabla_{w}\left(\frac{1}{N} \sum_{n=1}^{N} \ln p_{\text {pred }}\left(y_{n} \mid w\right)\right) .
$$

Further, if the observations are sampled from the ground truth distribution $q(y)$ and their number tends to infinity, then the GCP update (2.14) assumes the form

$$
\begin{aligned}
w_{\text {new }} & :=w-\lambda \cdot \mathbb{E}_{\mathbf{y} \sim q(y)}\left[\nabla_{w} K(w, \mathbf{y})\right]=w-\lambda \nabla_{w} \int \ln p_{\text {pred }}(y \mid w) q(y) d y \\
& =w-\lambda \nabla_{w} \int q(y) \ln \frac{q(y)}{p_{\text {pred }}(y \mid w)} d y=w-\lambda \nabla_{w} D_{\mathrm{KL}}\left(q \| p_{\text {pred }}(\cdot \mid w)\right) .
\end{aligned}
$$


Remark 2.1. $\quad$ 1. Formula 2.14) shows that the GCP update maximizes the likelihood of the predictive distribution $p_{\text {pred }}(y \mid w)$ for the observations $y_{1}, \ldots, y_{N}$.

2. Formula 2.15) shows that the GCP update is equivalent to the gradient descent step for the minimization of the $K L$ divergence from the ground truth distribution $q(y)$ to the predictive distribution $p_{\text {pred }}(y \mid w)$. If the ground truth distribution $q(y)$ belongs to the family $p_{\text {pred }}(y \mid w)$, then the minimum equals zero and is achieved for some (not necessarily unique) $w_{*}$ such that $p_{\text {pred }}\left(y ; w_{*}\right)=q(y)$; otherwise the minimum is positive and provides the best possible approximation of the ground truth in the sense of the KL divergence.

3. In our case, $q(y)$ is a normal distribution and $p_{\text {pred }}(y \mid w)$ are Student's t-distributions. In accordance with item 2, we will see below that the GCP update forces the number of degrees of freedom of $p_{\text {pred }}(y \mid w)$ to tend to infinity. However, due to the overparametrization of the predictive distribution (four parameters $m, \alpha, \beta, \nu$ instead of three), the learned variance of $q(y)$ will be represented by a curve in the space $(\beta, \nu)$. The limit point $\beta_{*}, \nu_{*}$ to which $\beta, \nu$ will converge during the GCP update, will depend on the initial condition. Interestingly, $\beta_{*}, \nu_{*}$ will always be different from the limit obtained by the classical CP update 2.7) (cf. Remark 3.4).

\subsection{Practical approaches}

Based on Remark 2.1 (items 1 and 2), we suggest the following general practical approach.

Practical approach 2.1. 1. One approximates the parameters of the prior by neural networks:

$$
m=m\left(x, w_{1}\right), \alpha=\alpha\left(x, w_{2}\right), \beta=\beta\left(x, w_{3}\right), \nu=\nu\left(x, w_{4}\right) .
$$

We call them the GCP neural networks.

2. One trains these four networks by the GCP update 2.10 until convergence of $m, \alpha, \beta, \nu$.

3. The resulting predictive distribution is the non-standardized Student's t-distribution $t_{2 \alpha}(y \mid m, \beta(\nu+1) /(\nu \alpha))$. The estimated mean $m_{\mathrm{est}}$ and variance $V_{\mathrm{est}}($ for $\alpha>1)$ are given by

$$
m_{\mathrm{est}}:=m, \quad V_{\mathrm{est}}:=\frac{\beta(\nu+1)}{(\alpha-1) \nu} .
$$

4. Student's $t$ variance $V_{\text {est }}$ overestimates the ground truth variance of the normal distribution $q(y)$. However, one can still recover the correct variance of $q(y)$ by appropriately correcting $V_{\mathrm{est}}$. We show that the correction is given by

$$
\tilde{V}_{\text {est }}:=\frac{\beta(\nu+1)}{(\alpha-A(\alpha)) \nu}
$$


with $A\left(\alpha_{*}\right)$ from Definition 3.1. We call it a correction formula for the variance. The interplay between $V_{\mathrm{est}}$ and $V_{\mathrm{est}}$ is illustrated in sections 5.1, 6.3, and 6.5. Our experiments show that this correction allows for reconstructing the ground truth variance even in the presence of outliers in the training set.

In the rest of the paper, we rigorously justify the above approach, study the dynamics of $m, \alpha, \beta, \nu$ under this update, and analyze how one should correct the variance for a fixed $\alpha$.

\section{Dynamics of $m, \alpha, \beta, \nu$}

\subsection{Dynamical system for $m, \alpha, \beta, \nu$}

The GCP update (2.10) induces the update for $(m, \alpha, \beta, \nu)$ as follows:

$$
m_{\text {new }}:=m\left(w-\lambda \cdot \nabla_{w} K\right)=m\left(w-\lambda \frac{\partial K}{\partial m} \nabla_{w} m(w)\right) \approx m(w)-\lambda\left(\nabla_{w} m\right)^{T}\left(\nabla_{w} m\right) \frac{\partial K}{\partial m},
$$

where $w=w_{1}$, and similarly for $\alpha, \beta, \nu$ and $w_{2}, w_{3}, w_{4}$, respectively.

Obviously, the new parameters $m_{\text {new }}, \alpha_{\text {new }}, \beta_{\text {new }}, \nu_{\text {new }}$ are different from $m^{\prime}, \alpha^{\prime}, \beta^{\prime}, \nu^{\prime}$ given by the classical conjugate prior update $(2.7)$. From now on, we replace $\lambda\left(\nabla_{w} m\right)^{T}\left(\nabla_{w} m\right)$, etc. by new learning rates and analyze how the parameters will change and to which values they will converge under the updates of the form

$$
m_{\text {new }}:=m-\lambda_{1} \frac{\partial K}{\partial m}, \quad \alpha_{\text {new }}:=\alpha-\lambda_{2} \frac{\partial K}{\partial \alpha}, \quad \beta_{\text {new }}:=\beta-\lambda_{3} \frac{\partial K}{\partial \beta}, \quad \nu_{\text {new }}:=\nu-\lambda_{4} \frac{\partial K}{\partial \nu},
$$

where $\lambda_{j}>0$ are the learning rates. As before, when we compute the derivatives of $K$, we keep all the prime-variables in (2.9) fixed and do not treat them as functions of $m, \nu, \alpha, \beta$. In other words, we first compute the derivatives of $K$ with respect to $m, \nu, \alpha, \beta$ and then substitute $m^{\prime}, \nu^{\prime}, \alpha^{\prime}, \beta^{\prime}$ from (2.7). For brevity, we will simply write $\partial K / \partial m$, etc. We call (3.2) the GCP update as well.

Setting

$$
\sigma:=\frac{\beta(\nu+1)}{\nu}
$$

we have

$$
\begin{aligned}
& \frac{\partial K}{\partial m}=\frac{\alpha+1 / 2}{\sigma+\frac{(m-y)^{2}}{2}}(m-y) \\
& \frac{\partial K}{\partial \alpha}=\ln \left(1+\frac{(m-y)^{2}}{2 \sigma}\right)+\Psi(\alpha)-\Psi\left(\alpha+\frac{1}{2}\right) \\
& \frac{\partial K}{\partial \beta}=\frac{1}{\beta}\left(\frac{\alpha+1 / 2}{1+\frac{(m-y)^{2}}{2 \sigma}}-\alpha\right) \\
& \frac{\partial K}{\partial \nu}=\frac{1}{2 \nu(\nu+1)}\left(\frac{\alpha+1 / 2}{\sigma+\frac{(m-y)^{2}}{2}}(m-y)^{2}-1\right)
\end{aligned}
$$




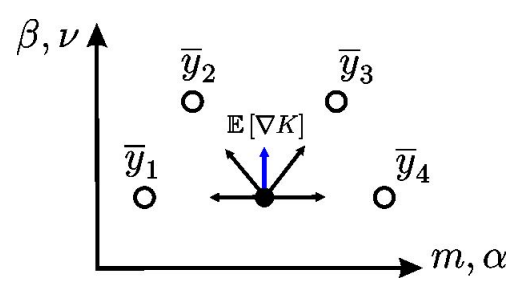

Figure 3.1: The black circle indicates the prior probability distribution 2.1) in the space of the parameters $m, \alpha, \beta, \nu$. The white circles indicate the posterior probability distributions (2.6) corresponding to different observations $y_{1}, y_{2}, \ldots$. The black vectors are the gradients with respect to the nonprime variables of the corresponding KL divergences. The blue vector is the averaged gradient. An equilibrium of system (3.8) would correspond to the case where the blue vector vanishes. Theorem 3.2 shows that this actually never happens. However, Theorem 4.1 shows that if one keeps $\alpha$ fixed, but updates $m, \beta, \nu$, then one obtains a whole curve of equilibria.

In this section and in the next one, we will treat the parameters $m, \alpha, \beta, \nu$ as functions of time $t>0$ and study a dynamical system that approximates the GCP update (3.2) when the number of observations is large. We will concentrate on the prototype situation, where all new learning rates are the same.

Condition 3.1. In the GCP update (3.2), we have $\lambda_{1}=\lambda_{2}=\lambda_{3}=\lambda_{4}$.

Under Condition 3.1, the approximating dynamical system takes the form

$$
\dot{m}=-\mathbb{E}\left[\frac{\partial K}{\partial m}\right], \quad \dot{\alpha}=-\mathbb{E}\left[\frac{\partial K}{\partial \alpha}\right], \quad \dot{\beta}=-\mathbb{E}\left[\frac{\partial K}{\partial \beta}\right], \quad \dot{\nu}=-\mathbb{E}\left[\frac{\partial K}{\partial \nu}\right] ;
$$

hereinafter the expectations are taken with respect to the true distribution $q(y)$ of $\mathbf{y}$ which is treated as a normally distributed random variable with mean $\mathbb{E}[\mathbf{y}]$ and variance $V:=\mathbb{V}[\mathbf{y}]$, see Fig. 3.1.

Remark 3.1. Due to (2.15), system (3.8) defines a gradient flow with the potential $D_{\mathrm{KL}}\left(q \| p_{\text {pred }}(\cdot \mid w)\right)$, where $\left.p_{\text {pred }}(y \mid w)\right)$ is the Student's t-distribution $t_{2 \alpha}(y \mid m, \beta(\nu+1) /(\nu \alpha))$.

Remark 3.2. If Condition 3.1 does not hold, then the respective factors $\lambda_{j}$ will appear in the right-hand sides in (3.8). The modifications one has to make in the arguments below are straightforward.

\subsection{Estimation of the mean $m$}

Using (3.4), we obtain the formula for the expectation

$$
\mathbb{E}\left[\frac{\partial K}{\partial m}\right]=\frac{\alpha+1 / 2}{(2 \pi V)^{1 / 2}} \int_{-\infty}^{\infty} \frac{m-y}{\sigma+\frac{(m-y)^{2}}{2}} e^{-\frac{(\mathbb{E}[y]-y)^{2}}{2 V}} d y .
$$


Theorem 3.1. The first equation in $(3.8)$ has a unique equilibrium $m=\mathbb{E}[\mathbf{y}]$. It is stable in the sense that, for any $\alpha, \beta, \nu$, we have

$$
\dot{m}<0 \text { if } m>\mathbb{E}[\mathbf{y}], \quad \dot{m}>0 \text { if } m<\mathbb{E}[\mathbf{y}] .
$$

Proof. Without loss of generality, assume that $\mathbb{E}[\mathbf{y}]=0$ and $V=1$ (otherwise, make a change of variables $z=(y-\mathbb{E}[\mathbf{y}]) / V^{1 / 2}$ in the integral in (3.9) $)$. Then we obtain from (3.9)

$$
\mathbb{E}\left[\frac{\partial K}{\partial m}\right]=C_{1} \int_{-\infty}^{\infty} \frac{m-y}{C_{2}+(m-y)^{2}} e^{-\frac{y^{2}}{2}} d y=-C_{1} \int_{-\infty}^{\infty} \frac{z}{C_{2}+z^{2}} e^{-\frac{(m+z)^{2}}{2}} d z,
$$

where $C_{1}, C_{2}>0$ do not depend on $m$.

Obviously, the right-hand side in (3.10) vanishes at $m=0$. Furthermore, due to (3.10), for $m>0$,

$$
\mathbb{E}\left[\frac{\partial K}{\partial m}\right]=C_{1} \int_{0}^{\infty} \frac{z}{C_{2}+z^{2}}\left(e^{-\frac{(m-z)^{2}}{2}}-e^{-\frac{(m+z)^{2}}{2}}\right) d z>0
$$

because $-(m-z)^{2}>-(m+z)^{2}$ for $m, z>0$. Similarly, $\mathbb{E}\left[\frac{\partial K}{\partial m}\right]<0$ for $m<0$.

\subsection{Estimation of the variance. The unbounded absorbing set}

From now on, taking into account Theorem 3.1, we assume the following.

Condition 3.2. $m=\mathbb{E}[\mathbf{y}]$.

Under Condition 3.2, we study the other three equations in (3.8), namely,

$$
\dot{\alpha}=-\mathbb{E}\left[\frac{\partial K}{\partial \alpha}\right], \quad \dot{\beta}=-\mathbb{E}\left[\frac{\partial K}{\partial \beta}\right], \quad \dot{\nu}=-\mathbb{E}\left[\frac{\partial K}{\partial \nu}\right],
$$

where (due to Condition 3.2)

$$
\begin{aligned}
& \mathbb{E}\left[\frac{\partial K}{\partial \alpha}\right]=\frac{1}{(2 \pi)^{1 / 2}} \int_{-\infty}^{\infty} \ln \left(1+\frac{V}{\sigma} \frac{z^{2}}{2}\right) e^{-\frac{z^{2}}{2}} d z+\Psi(\alpha)-\Psi\left(\alpha+\frac{1}{2}\right), \\
& \mathbb{E}\left[\frac{\partial K}{\partial \beta}\right]=\frac{1}{\beta}\left(\frac{\alpha+1 / 2}{(2 \pi)^{1 / 2}} \int_{-\infty}^{\infty} \frac{1}{1+\frac{V}{\sigma} \frac{z^{2}}{2}} e^{-\frac{z^{2}}{2}} d z-\alpha\right), \\
& \mathbb{E}\left[\frac{\partial K}{\partial \nu}\right]=\frac{1}{2 \nu(\nu+1)}\left(\frac{2 \alpha+1}{(2 \pi)^{1 / 2}} \int_{-\infty}^{\infty} \frac{z^{2}}{\frac{2 \sigma}{V}+z^{2}} e^{-\frac{z^{2}}{2}} d z-1\right) .
\end{aligned}
$$

\subsubsection{The functions $A(\alpha)$ and $\sigma_{\varkappa}(\alpha)$}

To formulate the main theorem of this section, we introduce a function $A(\alpha)$, which plays the central role throughout the paper. 


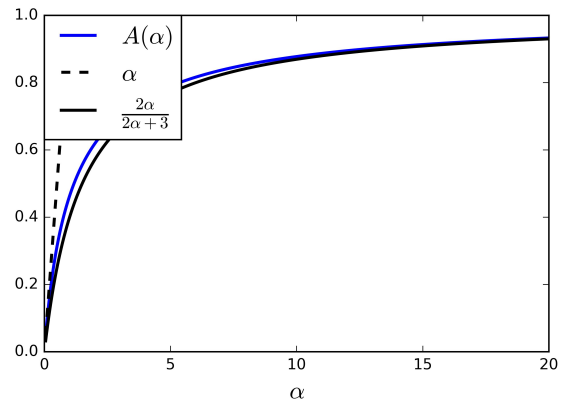

Figure 3.2: The function $A(\alpha)$ from Definition 3.1.

Definition 3.1. For each $\alpha>0, A=A(\alpha)$ is defined as a unique root of the equation

$$
F(\alpha-A)=\frac{\alpha}{(2 \alpha+1)(\alpha-A)}
$$

with respect to $A$, where

$$
F(x):=\frac{1}{(2 \pi)^{1 / 2}} \int_{-\infty}^{\infty} \frac{1}{2 x+z^{2}} e^{-\frac{z^{2}}{2}} d z \quad\left(=\frac{\pi^{1 / 2}}{2} \frac{e^{x} \operatorname{erfc}\left(x^{1 / 2}\right)}{x^{1 / 2}}\right), \quad x>0,
$$

and erfc is the complementary error function.

The main properties of $A(\alpha)$ are given in the following lemma (see Fig. 3.2).

Lemma 3.1. 1. Equation 3.15 has a unique root $A(\alpha)$,

2. $A(\alpha)$ is monotonically increasing,

3. $\frac{2 \alpha}{2 \alpha+3}<A(\alpha)<\min (\alpha, 1)$,

4. $A(\alpha)$ satisfies the differential equation

$$
A^{\prime}(\alpha)=1-\frac{2(\alpha-A(\alpha))}{(2 \alpha+1) A(\alpha)}
$$

5. $A(\alpha)$ has the following asymptotics:

$$
\begin{gathered}
A(\alpha)=\alpha-k_{0} \alpha^{2}+o\left(\alpha^{2}\right) \text { as } \alpha \rightarrow 0, \\
A(\alpha)=1-\frac{k_{1}}{\alpha}+o\left(\frac{1}{\alpha}\right) \text { as } \alpha \rightarrow \infty
\end{gathered}
$$

where $k_{0}=4 / \pi, k_{1}=3 / 2$.

Proof. These properties are proved in Lemmas A.1 A.4.

Definition 3.2. For each $\varkappa \geq 0$, we define the functions (see Fig. 3.3, left)

$$
\sigma_{\varkappa}(\alpha):=\left(1-\frac{\varkappa}{\alpha}\right)(\alpha-A(\alpha)) V, \quad \alpha>0 .
$$

We remind that $V=\mathbb{V}[\mathbf{y}]$. 


\subsubsection{Estimation of the variance}

The main result of this section (illustrated by Figures 3.3 and 3.4 ) is as follows.

Theorem 3.2. $\quad$ 1. There is a smooth increasing function $\sigma_{*}(\alpha), \alpha>0$, such that

(a) $\dot{\alpha}=0$ on the curve $\left(\alpha, \sigma_{*}(\alpha)\right)$,

(b) $\lim _{\alpha \rightarrow 0} \sigma_{*}(\alpha)=0$ and $\lim _{\alpha \rightarrow \infty} \sigma_{*}(\alpha)=\infty$,

(c) $\sigma_{*}(\alpha)<\sigma_{0}(\alpha)$ for all $\alpha>0$,

(d) for any $\varkappa>0$, there exists $\alpha_{\varkappa}>0$ such that

$$
\sigma_{*}(\alpha)>\sigma_{\varkappa}(\alpha) \text { for all } \alpha>\alpha_{\varkappa},
$$

(e) the region

$$
\mathbf{S}_{*}:=\left\{(\alpha, \beta, \nu) \in \mathbb{R}^{3}: \alpha, \beta, \nu>0 \text { and } \sigma_{*}(\alpha)<\frac{\beta(\nu+1)}{\nu}<\sigma_{0}(\alpha)\right\}
$$

is forward invariant for system (3.11.

2. For any $\alpha(0), \beta(0), \nu(0)>0$, there exists a time moment $t_{0}$ depending on the initial condition such that for all $t>t_{0},(\alpha(t), \beta(t), \nu(t)) \in \mathbf{S}_{*}, \dot{\alpha}(t), \dot{\beta}(t)>0, \dot{\nu}(t)<0$.

3. For any $\alpha(0), \beta(0), \nu(0)>0$, there is $C>0$ depending on the initial conditions such that the points $(\nu(t), \beta(t))$ for all $t \geq 0$ lie on the integral curve

$$
\beta^{2}+\nu^{2}+\frac{2 \nu^{3}}{3}=C
$$

of the equation

$$
\frac{d \beta}{d \nu}=-\frac{\nu(\nu+1)}{\beta}
$$

4. For any $\alpha(0), \beta(0), \nu(0)>0$, we have

$$
\alpha(t) \rightarrow \infty, A(\alpha(t)) \rightarrow 1, \nu(t) \rightarrow 0, \beta(t) \rightarrow \beta_{*} \quad \text { as } t \rightarrow \infty,
$$

where $\beta_{*}:=\left(\beta^{2}(0)+\nu^{2}(0)+\frac{2 \nu^{3}(0)}{3}\right)^{1 / 2}$.

Theorem 3.2 immediately implies the following corollary about the asymptotics of the variance $V_{\text {est }}$ in 2.4 for the predictive Student's t-distribution.

Corollary 3.1. For any $\alpha(0), \beta(0), \nu(0)>0$, we have

$$
\left(1-\frac{\varkappa}{\alpha}\right) \frac{\alpha-A(\alpha)}{\alpha-1}<\frac{V_{\text {est }}}{V}<\frac{\alpha-A(\alpha)}{\alpha-1} \quad \text { for all large enough } t \text {. }
$$

In particular,

$$
V_{\mathrm{est}} \rightarrow V \quad \text { as } t \rightarrow \infty
$$



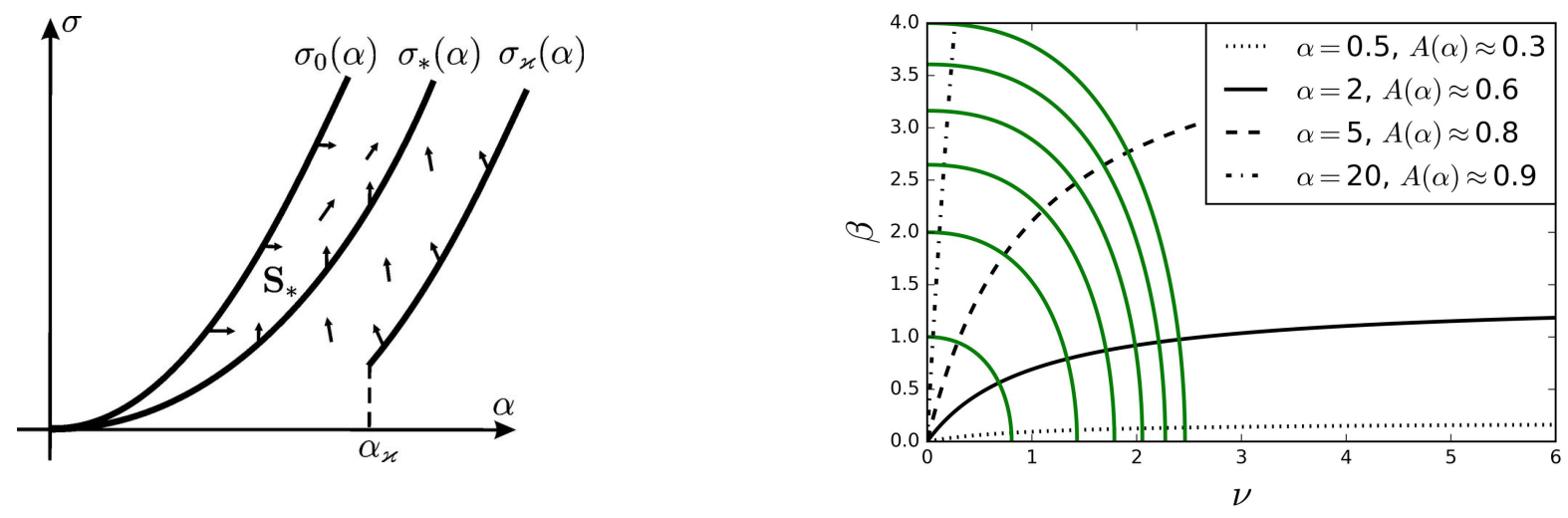

Figure 3.3: Left: The curves $\sigma_{0}(\alpha), \sigma_{\varkappa}(\alpha)$ given by (3.19), the curve $\sigma_{*}(\alpha)$ from Theorem 3.2. item 1 , and the region $\mathbf{S}_{*}$ given by $(3.20)$. The arrows indicate the directions of the vector field. Right: Green lines are the curves given by (3.21) for $C=1,4,7,10,13,16$. Black lines are the curves $\mathbf{C}_{\alpha, V}$ given by $\frac{\beta(\nu+1)}{\nu}=(\alpha-A(\alpha)) V$ with $V=1$. The black and green curves are orthogonal to each other.
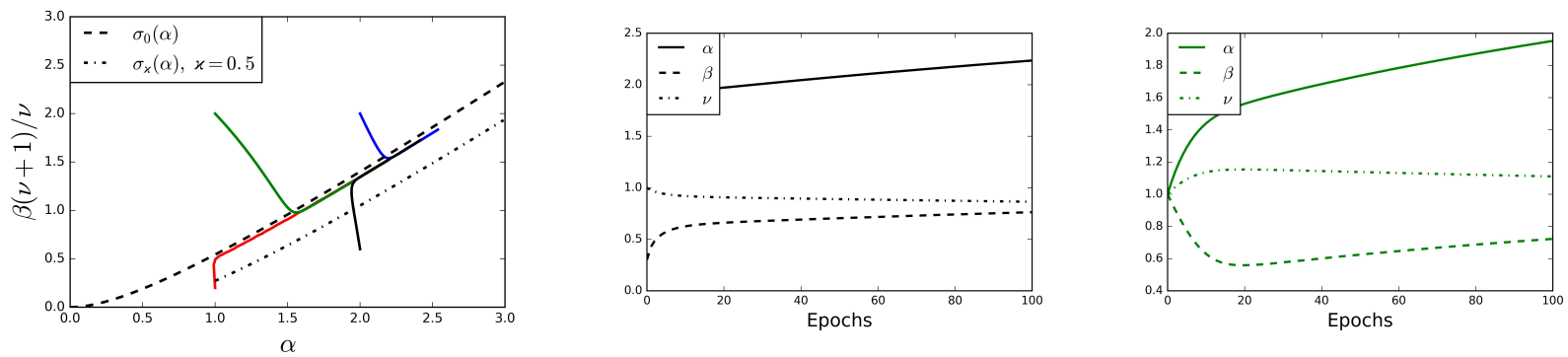

Figure 3.4: Left: Several trajectories obtained via iterating (3.2) for 2000 samples drawn from the normal distribution with mean 0 and variance 1. Middle/Right: Graphs of $\alpha, \beta, \nu$ plotted versus the number of epochs, corresponding to the lower-right/upper-left trajectory in the left figure.

Proof. From Theorem [3.2, item 1, we have by definition of $S_{*}$

$$
\left(1-\frac{\varkappa}{\alpha}\right)(\alpha-A(\alpha)) V<\frac{\beta(\nu+1)}{\nu}<(\alpha-A(\alpha)) V \text { for all sufficiently large } t .
$$

Deviding these inequalities by $\alpha-1$ and recalling that $\alpha(t) \rightarrow \infty$ as $t \rightarrow \infty$ and $A(\alpha) \rightarrow 1$ as $\alpha \rightarrow \infty$, we obtain the desired result.

Remark 3.3. One can show that $\varkappa / \alpha$ in the definition of the function $\sigma_{\varkappa}(\alpha)$ can be replaced by $\tilde{\varkappa} / \alpha^{2}$ with a sufficiently large $\tilde{\varkappa}$. In particular, the asymptotics in Corollary 3.1 will assume the form

$$
\left|\frac{V_{\text {est }}}{V}-1\right|=O\left(\alpha^{-2}\right) \quad \text { as } t \rightarrow \infty .
$$


The proof would require obtaining an extra term in the asymptotics of $A(\alpha)$ as $\alpha \rightarrow \infty$. However, we will not elaborate on these details.

Remark 3.4. Suppose the number of observations tends to infinity. Then in the standard conjugate prior update (2.7), the parameters $\alpha, \beta, \nu$ tend to infinity and the estimated mean and variance given by $(2.4)$ converge to the ground truth mean $\mathbb{E}[\mathbf{y}]$ and variance $V=\mathbb{V}[\mathbf{y}]$, while

$$
\mathbb{E}[\boldsymbol{\mu}] \rightarrow \mathbb{E}[\mathbf{y}], \quad \mathbb{V}[\boldsymbol{\mu}] \rightarrow 0, \quad \mathbb{E}[\boldsymbol{\tau}] \rightarrow \frac{1}{\mathbb{V}[\mathbf{y}]}, \quad \mathbb{V}[\boldsymbol{\tau}] \rightarrow 0
$$

The situation is quite different in Theorem 3.2. Although the parameter $\alpha$ tends to infinity, $\beta$ converges to a finite positive value and $\nu$ converges to zero. Nevertheless, the estimated variance $V_{\text {est }}$ in Corollary 3.1 converges to the ground truth variance $V=\mathbb{V}[\mathbf{y}]$, while (due to (2.2), 2.3), and (2.4)

$$
\mathbb{E}[\boldsymbol{\mu}] \rightarrow \mathbb{E}[\mathbf{y}], \quad \mathbb{V}[\boldsymbol{\mu}] \rightarrow \mathbb{V}[\mathbf{y}], \quad \mathbb{E}[\boldsymbol{\tau}] \rightarrow \infty, \quad \mathbb{V}[\boldsymbol{\tau}] \rightarrow \infty
$$

\subsection{Dynamics of $\alpha, \beta, \nu$. Proof of Theorem 3.2}

First, we show that $\mathbb{E}\left[\frac{\partial K}{\partial \beta}\right]$ and $\mathbb{E}\left[\frac{\partial K}{\partial \nu}\right]$ simultaneously vanish on the two-dimensional manifold

$$
\left\{(\alpha, \beta, \nu) \in \mathbb{R}^{3}: \alpha, \beta, \nu>0 \text { and } \frac{\beta(\nu+1)}{\nu}=\sigma_{0}(\alpha)\right\},
$$

where $\sigma_{0}(\alpha)$ is defined in $(3.19)$ Note that this manifold corresponds to the curve $\sigma=\sigma_{0}(\alpha)$ in Fig. 3.3 , left. We will also see that $\dot{\sigma}, \dot{\beta}>0$ and $\dot{\nu}<0$ in $\mathbf{S}_{*}$.

Lemma 3.2. We have

$$
\begin{gathered}
\dot{\beta}=\dot{\nu}=0 \quad \text { if } \sigma=\sigma_{0}(\alpha), \\
\dot{\sigma}, \dot{\beta}<0, \dot{\nu}>0 \quad \text { if } \sigma>\sigma_{0}(\alpha), \\
\dot{\sigma}, \dot{\beta}>0, \dot{\nu}<0 \quad \text { if } \sigma<\sigma_{0}(\alpha) .
\end{gathered}
$$

Proof. This lemma is proved in Appendix B.

Now we show that the trajectories $(\nu(t), \beta(t))$ lie on curves that do not depend on $\alpha$ or $V$, see the green lines in Fig. 3.3 (right).

Lemma 3.3. Let $\beta(t), \nu(t)(t>0)$ satisfy the last two equations in (3.11) (for an arbitrary $\alpha(t)>0)$. Then there is $C>0$ such that all the points $(\nu(t), \beta(t))$ belong to the integral curve 3.21 of the equation 3.22.

Proof. This lemma is proved in Appendix C.

Now we show that $\mathbb{E}\left[\frac{\partial K}{\partial \alpha}\right]$ is strictly negative on the manifold $(3.23)$, and, hence, neither system (3.8), nor system (3.11) possesses an equilibrium. 
Lemma 3.4. We have

$$
\dot{\alpha}>0 \quad \text { if } \sigma=\sigma_{0}(\alpha), \alpha>0 .
$$

Moreover, for any $\varkappa>0$, there exists $\alpha_{\varkappa}>0$ such that

$$
\dot{\alpha}<0 \quad \text { if } \sigma=\sigma_{\varkappa}(\alpha), \alpha>\alpha_{\varkappa} .
$$

Proof. This lemma is proved in Appendix D.

Proof of Theorem 3.2. The arguments below are illustrated by Fig. 3.3 .

Item 1. Note that, for each fixed $\sigma$, the function $\mathbb{E}\left[\frac{\partial K}{\partial \alpha}\right]$ is monotonically decreasing in $\alpha$. Furthermore by Lemma 3.4 we have $\dot{\alpha}>0$ on the curve $\sigma=\sigma_{0}(\alpha)$. On the other hand, $\dot{\alpha}<0$ for large $\alpha$, since $\Psi(\alpha)-\Psi(\alpha+1 / 2) \rightarrow 0$ as $\alpha \rightarrow \infty$. Thus, for each fixed $\sigma$ there exists a unique value $\alpha_{*}(\sigma)$ such that $\dot{\alpha}=0$. Moreover, since $\mathbb{E}\left[\frac{\partial K}{\partial \alpha}\right]$ depends monotonically on $\sigma$ and $\alpha$, the function $\alpha_{*}(\sigma)$ is smooth and can be inverted to a function $\sigma_{*}(\alpha)$ by the inverse function theorem. By construction, $\sigma_{*}(\alpha)$ satisfies all the properties in Theorem 3.2 . item 1 .

Item 2. We argue by contradiction. Suppose $(\alpha(t), \beta(t), \nu(t)) \notin \mathbf{S}_{*}$ for all $t \geq 0$. Then $\alpha(t)$ either decreases for all $t \geq 0$ or increases for all $t \geq 0$, since the trajectory cannot cross the manifold $\sigma=\sigma_{*}(\alpha)$. Suppose that it decreases. Since $\sigma_{*}(\alpha)$ increases in $\alpha$, it follows that $\sigma(t)$ remains bounded. Furthermore, by Lemma 3.2, $\sigma(t)$ increases. Hence, there exists $(\hat{\alpha}, \hat{\sigma})$ such that

$$
\lim _{t \rightarrow \infty}(\alpha(t), \sigma(t))=(\hat{\alpha}, \hat{\sigma}), \quad \lim _{t \rightarrow \infty}(\dot{\alpha}(t), \dot{\sigma}(t))=(0,0) .
$$

In particular, $\mathbb{E}\left[\frac{\partial K}{\partial \alpha}\right]$ has to vanish at $(\hat{\alpha}, \hat{\sigma})$. This may happen only if $(\hat{\alpha}, \hat{\sigma})$ belongs to the curve $\sigma=\sigma_{*}(\alpha)$. However, due to Lemma 3.2, $\lim _{t \rightarrow \infty} \dot{\sigma}(t)$ cannot vanish in this case, which is a contradiction with (3.28). A similar argument applies if $\alpha(t)$ increases.

Item 3 follows from Lemma 3.3 .

Item 4. Due to item 2, we can assume that $t_{0}=0$, so that $(\alpha(0), \beta(0), \nu(0)) \in \mathbf{S}_{*}$. By Lemma 3.2, $\alpha(t)$ and $\beta(t)$ are increasing, while $\nu(t)$ is decreasing. Furthermore, by Lemma 3.3. $\beta(t)$ is bounded for all $t$. Let us show that $\nu(t) \rightarrow 0$ as $t \rightarrow \infty$. Since the right-hand side in (3.14) has a singularity only for $\nu=0$, it remains to exclude the following two cases.

Case 1: $\nu(t) \rightarrow \tilde{\nu}$ as $t \rightarrow \infty$ for some $\tilde{\nu}>0$. In this case, $\beta(t) \rightarrow \tilde{\beta}$ for some finite $\tilde{\beta}>0$, and hence $\alpha(t) \rightarrow \tilde{\alpha}$ for some finite $\tilde{\alpha}>0$ since the trajectory must stay in $\mathbf{S}_{*}$. Therefore, $(\tilde{\alpha}, \tilde{\beta}, \tilde{\nu})$ must be an equilibrium of system (3.11). This contradicts Lemmas 3.2 and 3.4 .

Case 2: $\nu(t) \rightarrow 0$ as $t \rightarrow T$ for some finite $T>0$. In this case, $\beta(t) \rightarrow \tilde{\beta}$ as $t \rightarrow T$ for $\tilde{\beta}>0$ and hence $\alpha(t) \rightarrow \infty$ as $t \rightarrow T$ since the trajectory must stay in $\mathbf{S}_{*}$. But this is possible only if $\mathbb{E}\left[\frac{\partial K}{\partial \alpha}\right]$ is unbounded as $\alpha, \sigma \rightarrow \infty$, which is not the case due to (3.12).

By having excluded Cases 1 and 2 , we see that $\nu(t) \rightarrow 0$ as $t \rightarrow \infty$. Then $\beta(t) \rightarrow \tilde{\beta}$, where $\tilde{\beta}:=\left(\beta^{2}(0)+\nu^{2}(0)+\frac{2 \nu^{3}(0)}{3}\right)^{1 / 2}$ by to Lemma 3.3. Hence, $\sigma(t)=\frac{\beta(t)(\nu(t)+1)}{\nu(t)} \rightarrow \infty$. As the trajectory must stay in $\mathbf{S}_{*}$, it follows that $\alpha(t) \rightarrow \infty$. Finally, by Lemma 3.1 . $A(\alpha(t)) \rightarrow 1$. 


\section{Dynamics of $m, \beta, \nu$ for a fixed $\alpha$}

\subsection{Estimation of the variance. The curves of equilibria}

According to Theorem 3.2, neither system (3.8), nor system (3.11) possesses an equilibrium. However, this is not the case any more if the training dataset contains output outliers. Experiments show that $\alpha$ then converges to a finite value. In this section, we analyze the dynamics $m, \beta, \nu$ in $(3.2)$ for a fixed $\alpha$. We consider the update

$$
m_{\text {new }}:=m-\lambda \frac{\partial K}{\partial m}, \quad \beta_{\text {new }}:=\beta-\lambda \frac{\partial K}{\partial \beta}, \quad \nu_{\text {new }}:=\nu-\lambda \frac{\partial K}{\partial \nu} .
$$

As in Sec. 3, taking into account Theorem 3.1, we assume that the mean $m$ has already been learned: $m=\mathbb{E}[\mathbf{y}]$ (Condition 3.2). Then the corresponding approximating dynamical system is given by the two equations for $\beta, \nu$ from (3.8):

$$
\dot{\beta}=-\mathbb{E}\left[\frac{\partial K}{\partial \beta}\right], \quad \dot{\nu}=-\mathbb{E}\left[\frac{\partial K}{\partial \nu}\right],
$$

where the right-hand sides are explicitly given by (3.13) and (3.14). We consider this system on the quadrant $\left\{(\nu, \beta) \in \mathbb{R}^{2}: \nu, \beta>0\right\}$. Due to (3.25), this quadrant is forward invariant.

Remark 4.1. As in Remark 3.1, we conclude from 2.15) that system 4.2) defines the gradient flow with the potential $D_{\mathrm{KL}}\left(q \| p_{\text {pred }}(\cdot \mid w)\right)$, where $\left.p_{\text {pred }}(\cdot \mid w)\right)$ is the probability distribution of the Student's t-distribution $t_{2 \alpha}(y \mid \mathbb{E}[\mathbf{y}], \beta(\nu+1) /(\nu \alpha))$.

Theorem 4.1. Let $\alpha>0$ be fixed. Then the following hold.

1. Dynamical system (4.2) possesses a globally attracting family of equilibria lying on the curve

$$
\mathbf{C}_{\alpha, V}:=\left\{(\nu, \beta) \in \mathbb{R}^{2}: \nu>0, \frac{\beta(\nu+1)}{\nu}=\sigma_{0}(\alpha)\right\},
$$

where $\sigma_{0}(\alpha)$ is defined in (3.19).

2. Each trajectory $(\nu(t), \beta(t))$ lies on one of the integral curves (3.21). If $(\nu(0), \beta(0))$ lies below the curve $\mathbf{C}_{\alpha, V}$, then $\nu(t)$ decreases and converges to $\nu_{*}$ and $\beta(t)$ increases and converges to $\beta_{*}$. If $(\nu(0), \beta(0))$ lies above the curve $\mathbf{C}_{\alpha, V}$, then $\nu(t)$ increases and converges to $\nu_{*}$ and $\beta(t)$ decreases and converges to $\beta_{*}$. In both cases, $\left(\nu_{*}, \beta_{*}\right)$ is the point of intersection of the corresponding integral curve and the curve of equilibria $\mathbf{C}_{\alpha, V}$, see Fig. 3.3 .

3. The family of integral curves (3.21) is orthogonal to the family of the curves of equilibria $\left\{\mathbf{C}_{\alpha, V}\right\}_{\alpha, V>0}$. 

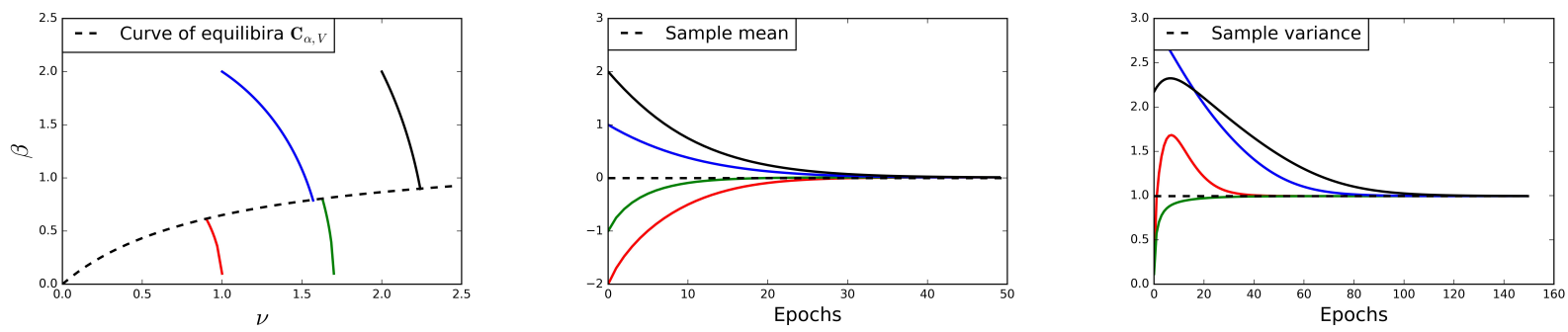

Figure 4.1: Several trajectories obtained via iterating (4.1) with 2000 samples drawn from the normal distribution with mean 0 and variance 1 . The parameter $\alpha$ is fixed: $\alpha=2$, $A(\alpha) \approx 0.619$. Left: plane $(\nu, \beta)$, with the initial condition $m(0)=0$ for all trajectories. Middle and Right: means $m$ and variances $\tilde{V}_{\text {est }}$ plotted versus the number of epochs. The initial conditions for $\beta$ and $\nu$ are the same as for the trajectories of the respective colors in the left plot, while $m(0)=-2,-1,1,2$.

Proof. Lemmas 3.2 and 3.3 imply items 1 and 2 . Let us prove item 3 . Assume that $(\nu, \beta)$ is a point of intersection of the curves

$$
\begin{aligned}
& \beta=f(\nu):=(\alpha-A) V \frac{\nu}{\nu+1} \quad \text { (a curve of equilibria), } \\
& \beta=g(\nu):=\left(C-\nu^{2}-\frac{2 \nu^{3}}{3}\right)^{1 / 2} \quad \text { (an integral curve). }
\end{aligned}
$$

Then $f^{\prime}(\nu)=\frac{(\alpha-A) V}{(\nu+1)^{2}}=\frac{\beta}{\nu(\nu+1)}$. On the other hand, by Lemma 3.3. $g^{\prime}(\nu)=-\frac{\nu(\nu+1)}{\beta}$. Thus, $f^{\prime}(\nu) g^{\prime}(\nu)=-1$, which implies the orthogonality of the two curves in 4.4.

Theorem 4.1 immediately implies the following corollary.

Corollary 4.1. Let $\alpha>0$ be fixed. Then for any $\beta(0), \nu(0)>0$, we have

$$
\tilde{V}_{\mathrm{est}}(t):=\frac{\beta(t)(\nu(t)+1)}{(\alpha-A(\alpha)) \nu(t)} \rightarrow V \quad \text { as } t \rightarrow \infty
$$

Figure 3.3 (right) shows the mutual configuration of the integral curves (3.21) and the curves of equilibria $\mathbf{C}_{\alpha, V}$ corresponding to different $\alpha$. Figure 4.1 (left) shows several trajectories in the $(\nu, \beta)$ plane converging to the curve of equilibria $\mathbf{C}_{\alpha, V}$. Figure 4.1 (middle and right) shows that taking initial conditions with $m(0) \neq \mathbb{E}[\mathbf{y}]$ still yields the proper convergence of the estimated mean $m_{\text {est }}:=m$ and the estimated variance $\tilde{V}_{\text {est }}$ in 4.5 .

Remark 4.2. Due to Theorem 4.1, each trajectory $(\nu(t), \beta(t))$ of system 4.2 can be obtained by solving the scalar differential equation

$$
\dot{\nu}=-\left.\mathbb{E}\left[\frac{\partial K}{\partial \nu}\right]\right|_{\beta=g(\nu)},
$$

where $g(\nu)$ is defined in (4.4) with a fixed $C>0$ (uniquely determined by $\nu(0), \beta(0)$ ). 


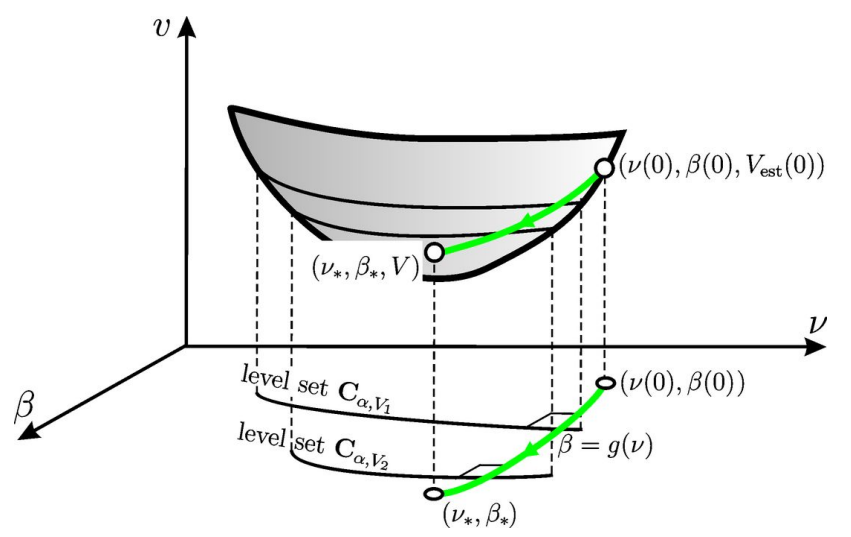

Figure 4.2: Schematic surface (4.7), its level sets $\mathbf{C}_{\alpha, V_{1}}$ and $\mathbf{C}_{\alpha, V_{2}}$, and the trajectory (in green) connecting the initial point $\left(\nu(0), \beta(0), V_{\text {est }}(0)\right)$ and the target point $\left(\nu_{*}, \beta_{*}, V\right)$. The projection $\beta=g(\nu)$ of the trajectory is orthogonal to the level sets, i.e., the trajectory follows the gradient descent on the surface 4.7).

Furthermore, one can use other functions $\tilde{g}(\nu)$ in 4.6 instead of $g(\nu)$. Due to (3.24), the resulting ODE would still have an equilibrium $\nu_{*}$ such that $\left(\nu_{*}, \tilde{g}\left(\nu_{*}\right)\right) \in \mathbf{C}_{\alpha, V}$, and at this equilibrium, we would have

$$
V=\frac{\beta_{*}\left(\nu_{*}+1\right)}{(\alpha-A(\alpha)) \nu_{*}}, \quad \beta_{*}:=\tilde{g}\left(\nu_{*}\right)
$$

One can also show that this equilibrium is globally stable for a broad class of functions $\tilde{g}(\nu)$.

However, the function $g(\nu)$ from (4.4), corresponding to the integral curve (3.21), is optimal in the following sense, see Fig. 4.2. Consider the two-dimensional surface in $\mathbb{R}^{3}$

$$
\left\{(\nu, \beta, v) \in \mathbb{R}^{3}: v=\frac{\beta(\nu+1)}{(\alpha-A(\alpha)) \nu}\right\}
$$

(with $\alpha$ fixed). Then the initial point $\left(\nu(0), \beta(0), \tilde{V}_{\text {est }}(0)\right)$ (where $\tilde{V}_{\text {est }}(t)$ is defined in (4.5)) and the target point $\left(\nu_{*}, \beta_{*}, V\right)$ (where $\nu_{*}, \beta_{*}$ are defined in Theorem 4.1, item 2) both lie on this surface. On the other hand, the curves $\left\{\mathbf{C}_{\alpha, V_{1}}\right\}_{V_{1}>0}$ are the level sets of this surface. Hence, due to Theorem 4.1, item 3, the curve $\beta=g(\nu)$ corresponds to the path of the gradient descent (or ascent) connecting the initial point $\left(\nu(0), \beta(0), V_{\text {est }}(0)\right)$ and the target point $\left(\nu_{*}, \beta_{*}, V\right)$.

Remark 4.3. It follows from Remark 4.1 and item 3 in Theorem 4.1 that, for any fixed $\alpha$ and $V=\mathbb{V}[\mathbf{y}]$, the curve $\mathbf{C}_{\alpha, V}$ is the set of minima of the potential $D_{\mathrm{KL}}\left(q \| p_{\text {pred }}(\cdot \mid w)\right)$, while all the other curves $\mathbf{C}_{\alpha_{1}, V_{1}}, \alpha_{1}, V_{1}>0$, are the level sets of this potential.

Remark 4.4. The situation in Theorem 4.1 is different both from the standard CP update (2.7) and from the GCP update (3.2) (cf. Remark 3.4). First, the parameter $\alpha$ is now fixed. Furthermore, each trajectory of system (4.2) (approximating the GCP update (4.1)) converges to a finite equilibrium $\left(\nu_{*}, \beta_{*}\right)$, where $\nu_{*}, \beta_{*}>0$. Nevertheless, the estimated variance $\tilde{V}_{\text {est }}$ given by $(4.5$ again converges to the ground truth variance $V=\mathbb{V}[\mathbf{y}]$. 

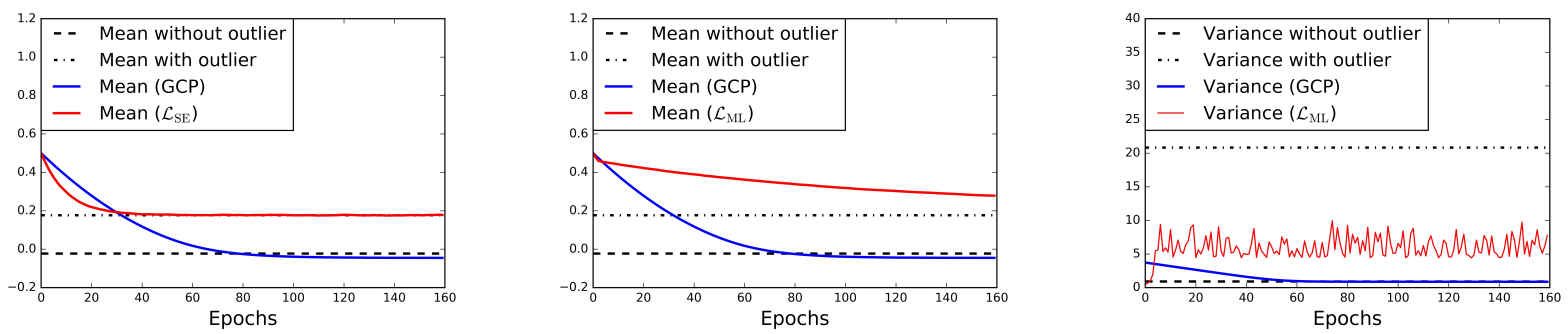

Figure 5.1: Fitting 500 samples drawn from the normal distribution with mean 0 and variance 1 and supplemented by an outlier $y=100$. Left: Means fitted with the GCP update (4.1) and, respectively, with the standard squared error loss $\mathcal{L}_{\mathrm{SE}}$. Middle/Right: Means/variances fitted with the GCP update (4.1) and, respectively, via maximizing the likelihood, i.e., minimizing $\mathcal{L}_{\mathrm{ML}}$. For the GCP update, the parameter $\alpha$ is fixed: $\alpha=1$, $A(\alpha) \approx 0.46$, and the variance is estimated by $\tilde{V}_{\text {est }}$ in 4.5 .

\section{$5 \quad$ Role of a fixed $\alpha$}

\subsection{Sensitivity to outliers}

It is well known that outliers essentially influence the estimate of the mean $m$ if one uses the standard squared error loss

$$
\mathcal{L}_{\mathrm{SE}}(y, m)=(m-y)^{2} .
$$

The same is true when one estimates both mean $m$ and precision $p$ via maximizing the log-likelihood of a normal distribution, or, equivalently, minimizing the loss

$$
\mathcal{L}_{\mathrm{ML}}(y, m, p)=p(m-y)^{2}-\ln p .
$$

The reason is that, in both cases, the derivatives of the loss functions $\mathcal{L}_{\mathrm{SE}}$ and $\mathcal{L}_{\mathrm{ML}}$ with respect to $m$ are proportional to $m-y$, while the derivative of $\mathcal{L}_{\mathrm{ML}}(y, m, p)$ with respect to $p$ contains even $(m-y)^{2}$. It turns out that the GCP update (4.1) is much less sensitive to outliers, see Fig. 5.1. This can be explained by the fact that the derivatives of the KL divergence with respect to $m, \beta$ and $\nu$ are bounded with respect to $m-y$, see (3.4), (3.6), and (3.7). Moreover, $\frac{\partial K}{\partial m}$ even vanishes as $m-y \rightarrow \infty$. Another explanation is that the GCP update is equivalent to maximizing the likelihood of the Student's t-distribution (item 1 in Remark 2.1). It is known [35] that the optimal value of $m$ is different from the sample mean due to downweighting the outlying observations. In sections 6.3 and 6.5 , we further analyze the performance of the GCP neural networks on contaminated data sets in comparison with other neural network methods.

\subsection{Learning speed in clean and noisy regions}

\subsubsection{Observations}

When one approximates the parameters $m, \beta, \nu$ by neural networks, one represents these parameters as functions of an input variable $x \in \mathbb{R}^{m}$ and of a set of weights $w \in \mathbb{R}^{M}$. Since 


\begin{tabular}{c|c|c}
$\alpha$ & LerSp $(m)$ & LerSp $($ Var $)$ \\
\hline Small & cl. $>$ noisy & cl. $>$ noisy \\
\hline Large & cl. $\gg$ noisy & cl. $<$ noisy
\end{tabular}

Table 5.1: Relative learning speed of estimated mean $m$ and variance $\tilde{V}_{\text {est }}$ for clean (cl.) and noisy regions. Notation " $<$ " and " $>$ " stands for a "lower" and a "higher" speed, and " $\gg$ " for a "much higher" speed.

neural networks have finite capacity ( $M$ is finite), they cannot perfectly approximate $m, \beta, \nu$ for all $x$ simultaneously. Therefore, it is important to understand in which regions of the input space $\mathbb{R}^{m}$ the parameters are approximated better and in which worse, cf. [11]. This is directly related to the values of the gradients in the GCP update (4.1), which determine the learning speed. The faster the learning in a certain region occurs, the more influential this region is. In particular, we are interested in the learning speed in so called clean regions (where $V$ is small) compared with noisy regions (where $V$ is large).

Below, we will concentrate on the regime where the learning process starts and the initial conditions for $\beta$ and $\nu$ satisfy

$$
\beta(0) \approx \nu(0) \approx 1
$$

This is often the case if $\beta$ and $\nu$ are approximated by neural networks with the softplus output, e.g.,

$$
\beta=\ln \left(1+e^{w}\right),
$$

where $w \in \mathbb{R}$ is the input of the softplus output.

In the observations below, we denote the learning speed of the mean and the variance by LerSp $(m)$ and LerSp(Var), respectively.

Observation 5.1. Let $\alpha$ be small.

1. In clean regions (small $V)$ : $\operatorname{LerSp}(m)$ is of order 1 and $\operatorname{LerSp}(\operatorname{Var})$ is of order 1 .

2. In noisy regions (large $V)$ : $\operatorname{LerSp}(m)$ is of order $1 / V$ and $\operatorname{LerSp}(\operatorname{Var})$ is of order $\alpha$.

Observation 5.2. Let $\alpha$ be large.

1. In clean regions $($ small $V): \operatorname{LerSp}(m)$ is of order $\alpha$ and $\operatorname{LerSp}(\operatorname{Var})$ is of order 1 .

2. In noisy regions (large $V)$ : $\operatorname{LerSp}(m)$ is of order $1 / V$ and $\operatorname{LerSp}(\operatorname{Var})$ is of order $\alpha$.

Observations 5.1 and 5.2 are summarized in Table 5.1. In particular, we see that the mean is always learned faster in clean regions. Taking large $\alpha$ further increases the learning speed of the mean in clean regions, but simultaneously increases the learning speed of variance in noisy regions compared with clean regions.

Observation 5.3. The values of $\beta_{*}$ and $\nu_{*}$ to which the trajectory of (4.2) will converge are determined by the value $(\alpha-A(\alpha)) V$. 

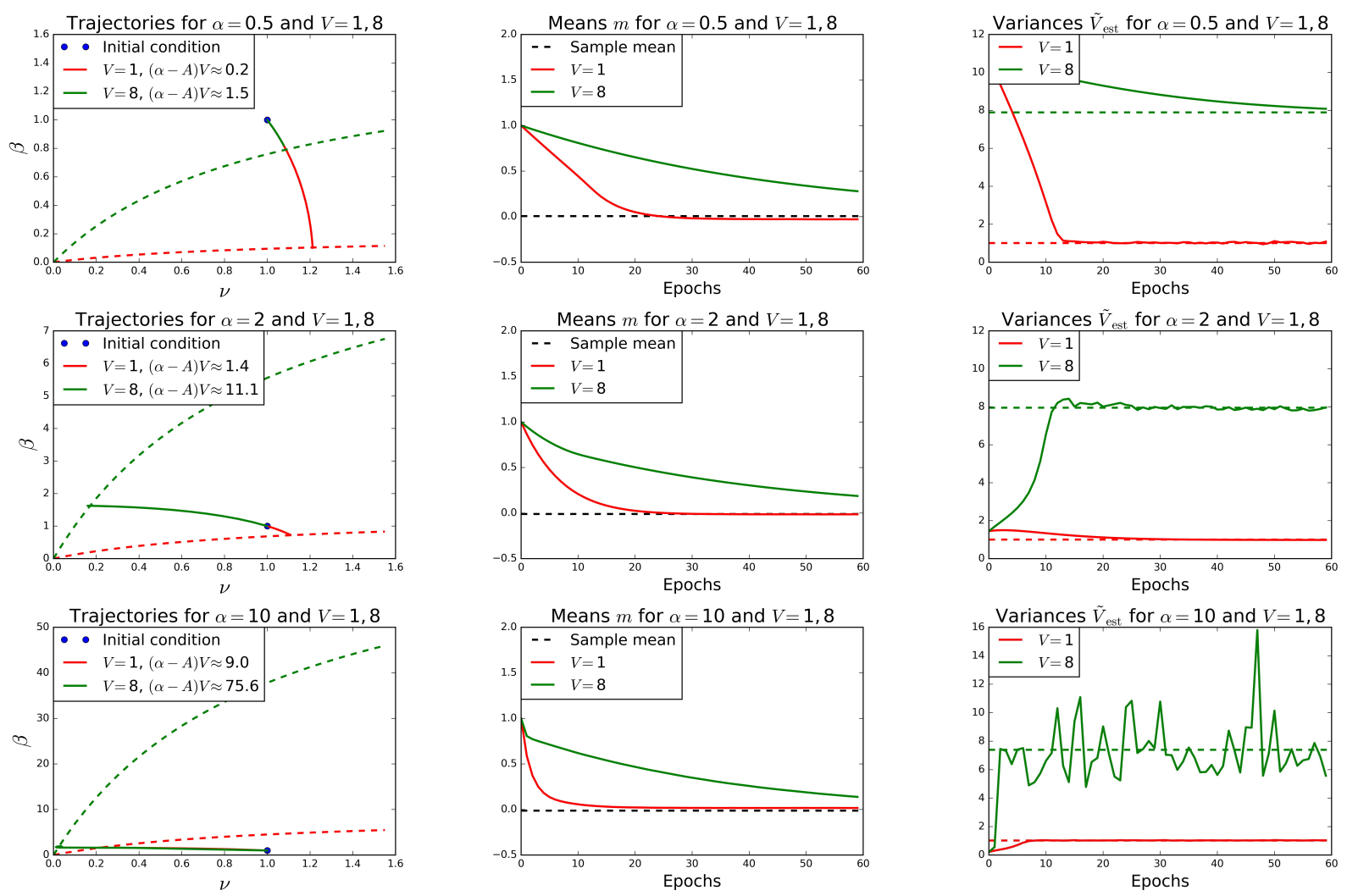

Figure 5.2: Trajectories (left) and graphs of means (middle) and variances (right) versus the number of epochs for different values of $\alpha$ and $V$, based on the GCP update (4.1) for 2000 samples drawn from the normal distribution with mean 0 and variance $V$. The initial conditions are $m(0)=\beta(0)=\nu(0)=1$. The dashed lines in the left-hand column indicate the corresponding curves of equilibria $\mathbf{C}_{\alpha, V}$. The dashed lines in the right-hand column indicate the corresponding sample variances.

1. If $(\alpha-A(\alpha)) V \ll 1$, then $\beta_{*} \approx 0$ and $\nu_{*} \approx 1$.

2. If $(\alpha-A(\alpha)) V \gg 1$, then $\beta_{*} \approx 1$ and $\nu_{*} \approx 0$.

Small values of $\beta$ and $\nu$ will lead to large gradients $\frac{\partial K}{\partial \beta}$ and $\frac{\partial K}{\partial \nu}$, respectively, which may cause large oscillations of $\tilde{V}_{\text {est }}$.

Observations 5.15 .3 are illustrated in Fig. 5.2 and explained in detail below.

\subsubsection{Justification of the observations}

1. First, we analyze LerSp(Var). Consider the limit $V \rightarrow 0$ (clean regions). Due to (4.3), the point $(\nu, \beta)$ lies above the line of equilibria $\mathbf{C}_{\alpha, V}$ at a vertical distance of order 1 from 
it. Using (3.13) and (3.14), we see that

$$
\lim _{V \rightarrow 0} \mathbb{E}\left[\frac{\partial K}{\partial \beta}\right]=\frac{1}{2 \beta}, \quad \lim _{V \rightarrow 0} \mathbb{E}\left[\frac{\partial K}{\partial \nu}\right]=-\frac{1}{2 \nu(\nu+1)},
$$

i.e. $(\nu, \beta)$ approaches $\mathbf{C}_{\alpha, V}$ with speed of order 1 .

Now consider the limit $V \rightarrow \infty$. Due to 4.3 , the point $(\nu, \beta)$ lies below and to the right from the curve of equilibria $\mathbf{C}_{\alpha, V}$ at a horizontal distance of order 1 from it. Using (3.13) and (3.14), we see that

$$
\lim _{V \rightarrow \infty} \mathbb{E}\left[\frac{\partial K}{\partial \beta}\right]=-\frac{\alpha}{\beta}, \quad \lim _{V \rightarrow \infty} \mathbb{E}\left[\frac{\partial K}{\partial \nu}\right]=\frac{\alpha}{\nu(\nu+1)},
$$

i.e. $(\nu, \beta)$ approaches $\mathbf{C}_{\alpha, V}$ with speed of order $\alpha$.

These arguments justify the assertions about LerSp(Var) in Observations 5.1 and 5.2 .

Further, recall that the trajectory $(\nu, \beta)$ lies on one of the curves (3.21). Thus, if $(\alpha-$ $A(\alpha)) V \ll 1$ and $(\nu, \beta)$ approaches $\mathbf{C}_{\alpha, V}$, the value of $\beta$ will approach 0 , while $\nu$ will stay of order 1. On the other hand, if $(\alpha-A(\alpha)) V \gg 1$ and $(\nu, \beta)$ approaches $\mathbf{C}_{\alpha, V}$, the value of $\beta$ will stay of order 1 and $\nu$ will approach 0 . This is illustrated in Fig. 5.2 (left-hand column). This justifies Observation 5.3 .

2. Now we analyze $\operatorname{LerSp}(m)$. Here we assume that the variance $V$ has already been estimated approximately. We express this fact by assuming that the parameters $\beta$ and $\nu$ are such that

$$
\tilde{V}_{\text {est }}=c V
$$

for some $c \in\left(c_{1}, c_{2}\right)$, where $c_{2}>c_{1}>0$ do not depend on $V$. Without loss of generality, assume that $\mathbb{E}[\mathbf{y}]=0$ and $m>0$. Then, due to (3.9),

$$
\begin{aligned}
\mathbb{E}\left[\frac{\partial K}{\partial m}\right] & =(\alpha+1 / 2) \frac{1}{(2 \pi)^{1 / 2}} \int_{-\infty}^{\infty} \frac{m-V^{1 / 2} z}{c(\alpha-A) V+\frac{\left(m-V^{1 / 2} z\right)^{2}}{2}} e^{-\frac{z^{2}}{2}} d z \\
& =(\alpha+1 / 2) \frac{1}{(2 \pi V)^{1 / 2}} \int_{-\infty}^{\infty} \frac{\frac{m}{V^{1 / 2}}-z}{c(\alpha-A)+\frac{\left(\frac{m}{V^{1 / 2}}-z\right)^{2}}{2}} e^{-\frac{z^{2}}{2}} d z .
\end{aligned}
$$

Hence,

$$
\begin{aligned}
& \mathbb{E}\left[\frac{\partial K}{\partial m}\right]=\frac{2 \alpha+1}{m}+o(V) \quad \text { as } V \rightarrow 0, \\
& \mathbb{E}\left[\frac{\partial K}{\partial m}\right]=\frac{k(\alpha)}{V}+o\left(\frac{1}{V}\right) \quad \text { as } V \rightarrow \infty,
\end{aligned}
$$

where

$$
k(\alpha)=\frac{(\alpha+1 / 2) m}{(2 \pi)^{1 / 2}} \int_{-\infty}^{\infty} \frac{c(\alpha-A)-z^{2} / 2}{\left(c(\alpha-A)+z^{2} / 2\right)^{2}} e^{-\frac{z^{2}}{2}} d z .
$$

The constant $k(\alpha)$ can be obtained by dividing the integral in the right-hand side of (5.4) by $V^{-1 / 2}$ and applying L'Hospital's rule. Using the properties of $A(\alpha)$ in (3.18), one can show that $k(\alpha)$ is positive, bounded, and bounded away from zero for all $\alpha>0$.

These arguments justify the assertions about $\operatorname{LerSp}(m)$ in Observations 5.1 and 5.2 . 


\section{GCP neural networks: experiments}

\subsection{Methods}

We compare the following methods:

1. the GCP method with and without the correction formula for $\tilde{V}_{\text {est }}$ in $(2.18)$. Whenever we apply the correction formula, we indicate this by writing $\mathrm{GCP}_{\text {corr }}$;

2. the maximum likelihood method (ML), in which one maximizes the likelihood of the normal distribution with the input-dependent mean and precision,

3. density power divergence method (DPD) [2,10], in which one minimizes the density power (instead of the KL) divergence from the ground truth distribution to the approximating normal distribution; as the GCP, this method is known to be robust against outliers,

4. Bayesian maximum likelihood method ( $\left.\mathrm{ML}_{\text {Bayes }}\right)[19]$, in which one maximizes the likelihood of the normal distribution with input-dependent precision, using dropout for both training and prediction to approximate the posterior distribution of the weights.

5. Stein variational gradient descent (SVGD) [28]; Bayesian method, in which we use a Gaussian likelihood with input-dependent mean and input-independent variance and a particle approximation of the posterior distribution of the weights,

6. the probabilistic back propagation (PBP) [13]; Bayesian method, in which the posterior of weights is approximated with assumed density filtering 38 and expectationpropagation [32] methods. The variance of the Gaussian likelihood is assumed inputindependent.

Note that the GCP $\left(\mathrm{GCP}_{\text {corr }}\right)$, ML, and DPD estimate aleatoric heteroscedastic uncertainty; the Bayesian methods SVGD and PBP estimate aleatoric homoscedastic and epistemic heteroscedastic uncertainty; and the $\mathrm{ML}_{\text {Bayes }}$ estimates both uncertainties as heteroscedastic.

\subsection{Synthetic data set: aleatoric vs. epistemic uncertainty}

We evaluate the predictive distributions in the above methods for a synthetic data set. The input data set $X$ consists of 20 points uniformly distributed on $[-4,-2] \cup[2,4]$. For each $x \in X$, we sample $y$ from the normal distribution with mean $x^{3}$ and standard deviation 3 . In this and next subsections, we use one-hidden layer networks with 100 hidden units. Figure6.1 confirms that the GCP ( $\left.\mathrm{GCP}_{\text {corr }}\right)$, ML, and DPD capture only aleatoric uncertainty, while the Bayesian methods $\mathrm{ML}_{\text {Bayes }}$, PBP, and SVGD also capture epistemic uncertainty due to the lack of data. To visualize the two types of uncertainty, we follow [5] and use the law of total variance $\mathbb{V}[\mathbf{y} \mid x]=\mathbb{E}_{w}[\mathbb{V}[\mathbf{y} \mid x, w]]+\mathbb{V}_{w}[\mathbb{E}[\mathbf{y} \mid x, w]]$, where the first term corresponds to aleatoric variance and the second term to epistemic. 

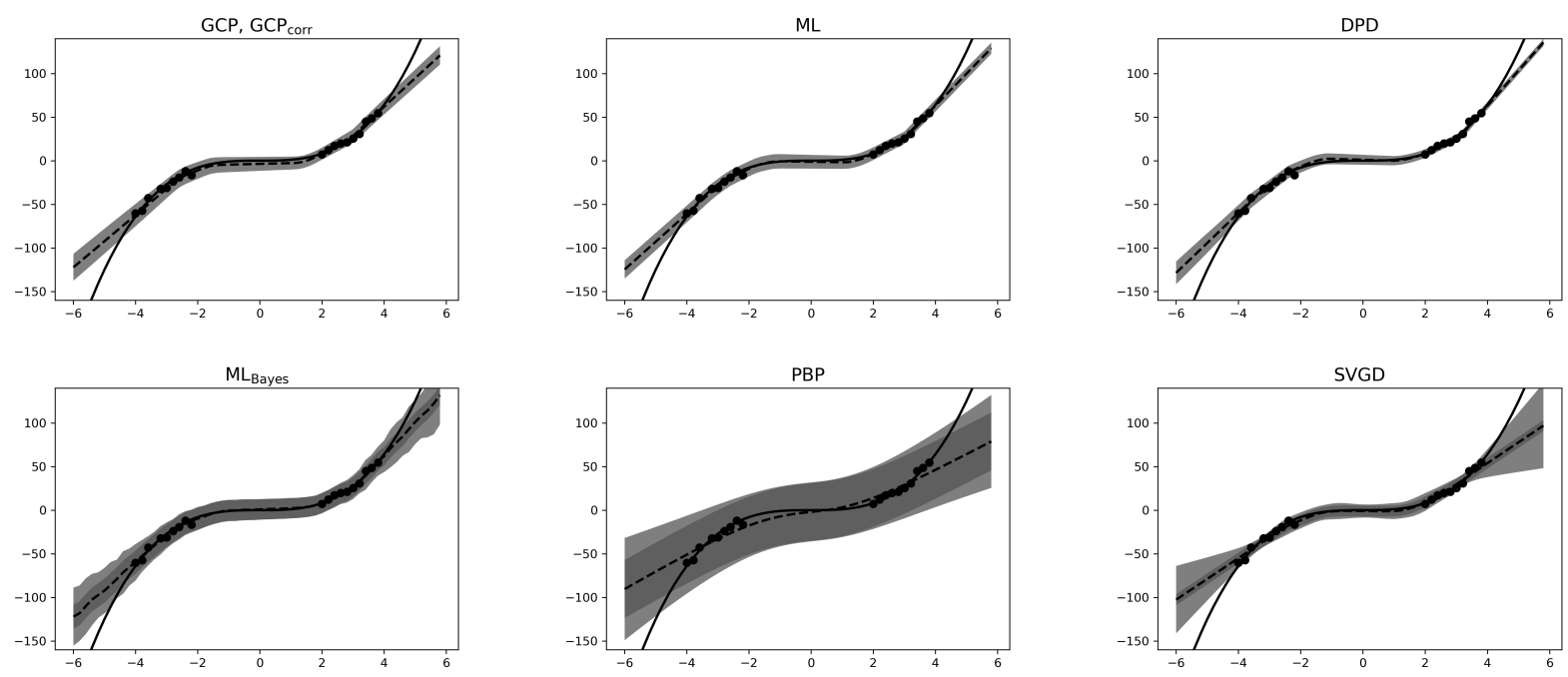

Figure 6.1: Predictions made by different methods on the synthetic data set $\mathbf{y} \sim \mathcal{N}\left(x^{3}, 3^{2}\right)$. The samples are shown as filled circles, the ground truth as a solid line, the predicted mean as a dashed line. For the Bayesian methods $\mathrm{ML}_{\text {Bayes }}$, PBP, and SVGD, the darker shaded areas correspond to \pm 3 aleatoric standard deviations and the lighter shaded areas correspond to \pm 3 standard deviations of the predictive distribution. For the non-Bayesian methods GCP $\left(\mathrm{GCP}_{\text {corr }}\right), \mathrm{ML}$, and $\mathrm{DPD}$, the aleatoric standard deviation coincides with the predictive one (both indicated as the lighter shaded areas). In the absence of outliers, GCP and GCP corr predict almost identical variance due to large values of $\alpha$.

\subsection{Synthetic data set: robustness to outliers in the training set}

We generate a synthetic data set containing $5 \%$ of outliers. The set $X$ consists of 400 points uniformly distributed on the interval $(-1,1)$. For each $x \in X$, with probability 0.95 we sample $y$ from the normal distribution with mean $\sin (3 x)$ and standard deviation $0.5 \cos ^{4} x$, and with probability 0.05 we sample $y$ from a uniform distribution on the interval $(-4,16)$. Figure 6.2 shows the data and the fits of different methods. The means predicted by the GCP and DPD are significantly less affected by the outliers compared with the other methods. However, the standard deviations $s^{3}$ predicted by all the methods, except for the $\mathrm{GCP}_{\text {corr }}$, are significantly distorted. Although the DPD is known to be robust against the outliers, it does not manage to properly capture the $x$-dependence of the variance. The ML, $\mathrm{ML}_{\text {Bayes }}$, SVGD, PBP, and the GCP without the correction overestimate the variance. However, using the correction formula for $\tilde{V}_{\text {est }}$ in $(2.18)$ allows $\mathrm{GCP}_{\text {corr }}$ to reconstruct the ground truth variance. Furthermore, the knowledge of $\alpha$ in the GCP provides additional information, namely, small values of $\alpha$ indicate that the corresponding samples belong to a (less trust-worthy) region in which the training set contained outliers.

\footnotetext{
${ }^{3}$ For Bayesian methods, we plot the standard deviations of the predictive distributions. The epistemic standard deviations are negligible in this example due to a relatively large amount of data.
} 

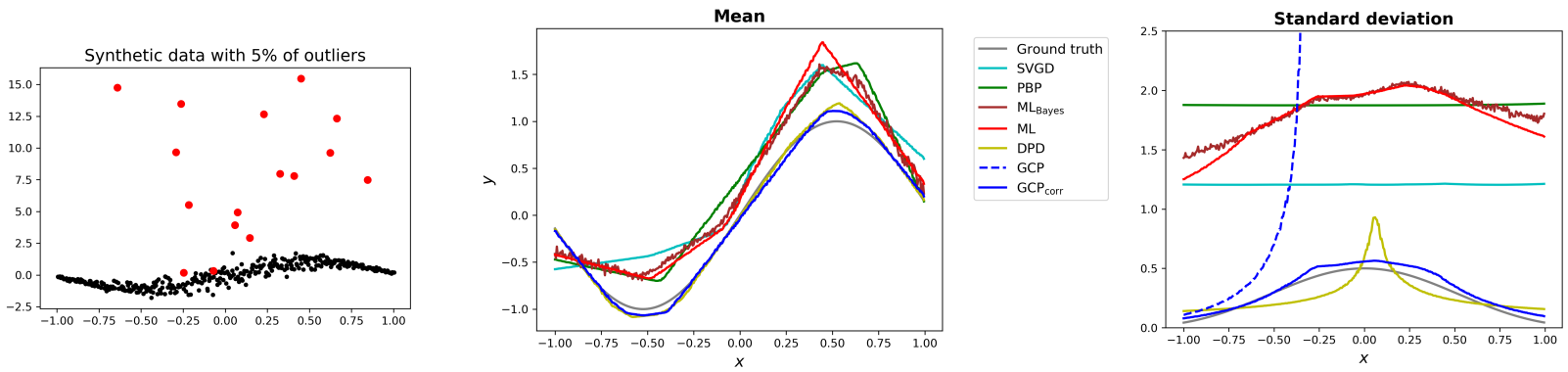

Figure 6.2: Left: Synthetic data with mean $\sin (3 x)$ and standard deviation $0.5 \cos ^{4} x$ (black dots), complemented by $5 \%$ of outliers sampled from a uniform distribution on the interval $(-4,16)$ (red disks). Middle: The ground truth mean $\sin (3 x)$ and the means predicted by the different methods. Right: The ground truth standard deviation $0.5 \cos ^{4} x$ and the standard deviations predicted by the different methods.

\subsection{Real world data sets}

Architectures. We use one-hidden layer networks for the parameters of the prior 2.16) with ReLU nonlinearities. Each network contains 50 hidden units for all the data sets below, except for the largest MSD set. For the latter, we use 100 hidden units. For regularization, we use a dropout layer between the hidden layer and the output unit. Our approach is directly applicable to neural networks of any depth and structure, however we kept one hidden layer for the compatibility of our validation with [11, 13, 23, 24].

Measures. We use two measures to estimate the quality of the fit.

1. The overall root mean squared error $(R M S E)$.

2. The area under the following curve $(A U C)$, measuring the trade-off between properly learning the mean and the variance. Assume the test set contains $N$ samples. We order them with respect to their predicted variance. For each $n=0, \ldots, N-1$, we remove $n$ samples with the highest variance and calculate the RMSE for the remaining $N-n$ samples (with the lowest variance). We denote it by $\operatorname{RMSE}(n)$ and plot it versus $n$ as a continuous piecewise linear curve. The second measure is the area under this curve normalized by $N-1$ :

$$
\mathrm{AUC}:=\frac{1}{N-1} \sum_{n=0}^{N-2} \frac{\operatorname{RMSE}(n)+\operatorname{RMSE}(n+1)}{2} .
$$

Data sets. We analyze the following publicly available data sets: Boston House Prices [12] (506 samples, 13 features), Concrete Compressive Strength [48] (1030 samples, 8 features), Combined Cycle Power Plant [18, 43] (9568 samples, 4 features), Yacht Hydrodynamics [9, 39] (308 samples, 6 features), Kinematics of an 8 Link Robot Arm Kin8Nm ${ }^{4}$ (8192 samples, 8

\footnotetext{
${ }^{4}$ http://mldata.org/repository/data/viewslug/regression-datasets-kin8nm/
} 


\begin{tabular}{|c|c|c|c|c|c|c|c|c|c|c|c|c|}
\hline \multicolumn{3}{|c|}{ Boston } & \multicolumn{2}{|l|}{ Concrete } & \multicolumn{2}{|l|}{ Power } & \multicolumn{2}{|l|}{ Yacht } & \multicolumn{2}{|l|}{ Kin8nm } & \multicolumn{2}{|l|}{ MSD } \\
\hline & RMSE & AUC & RMSE & AUC & RMSE & AUC & RMSE & AUC & RMSE & AUC & RMSE & AUC \\
\hline SVGD & $2.93 \pm 1.02$ & $2.21 \pm 0.61$ & $5.15 \pm 0.65$ & $.21 \pm 0.72$ & $4.13 \pm 0.32$ & $4.10 \pm 0.28$ & $0.88 \pm 0.40$ & $0.37 \pm 0.18$ & $0.09 \pm 0.01$ & $0.08 \pm 0.01$ & $8.93 \pm \mathrm{NA}$ & $8.25 \pm \mathrm{NA}$ \\
\hline PBP & $2.99 \pm 0.97$ & $2.10 \pm 0.47$ & $5.37 \pm 0.60$ & $4.45 \pm 0.56$ & $4.11 \pm 0.30$ & $3.85 \pm 0.23$ & $1.04 \pm 0.39$ & $0.57 \pm 0.13$ & $0.10 \pm 0.00$ & $0.08 \pm 0.00$ & $8.88 \pm \mathrm{NA}$ & $6.79 \pm \mathrm{NA}$ \\
\hline $\mathrm{ML}_{\text {Bayes }}$ & $3.55 \pm 1.41$ & $1.92 \pm 0.39$ & $5.45 \pm 0.63$ & $3.75 \pm 0.52$ & $4.19 \pm 0.30$ & $3.72 \pm 0.21$ & $0.78 \pm 0.39$ & $0.24 \pm 0.07$ & $0.11 \pm 0.01$ & $0.07 \pm 0.00$ & $8.90 \pm \mathrm{NA}$ & $5.19 \pm \mathrm{NA}$ \\
\hline ML & $3.63 \pm 1.49$ & $2.00 \pm 0.48$ & $5.29 \pm 0.81$ & $3.51 \pm 0.59$ & $4.15 \pm 0.30$ & $3.70 \pm 0.24$ & $0.82 \pm 0.41$ & $0.25 \pm 0.09$ & $0.10 \pm 0.01$ & $0.06 \pm 0.00$ & $8.91 \pm \mathrm{NA}$ & $5.22 \pm \mathrm{NA}$ \\
\hline DPD & $3.91 \pm 1.74$ & $2.21 \pm 0.63$ & $6.05 \pm 0.86$ & $4.23 \pm 0.83$ & $4.12 \pm 0.32$ & $3.66 \pm 0.36$ & $2.48 \pm 1.24$ & $0.36 \pm 0.16$ & $0.17 \pm 0.02$ & $0.07 \pm 0.00$ & $9.91 \pm \mathrm{NA}$ & $6.06 \pm \mathrm{NA}$ \\
\hline GCP & $3.74 \pm 1.59$ & $1.79 \pm 0.40$ & $5.47 \pm 0.65$ & $3.53 \pm 0.65$ & $4.13 \pm 0.31$ & $3.54 \pm 0.33$ & $0.96 \pm 0.49$ & $0.23 \pm 0.09$ & $0.09 \pm 0.01$ & $0.06 \pm 0.00$ & $9.23 \pm \mathrm{NA}$ & $5.24 \pm \mathrm{NA}$ \\
\hline
\end{tabular}

Table 6.1: RMSE and AUC for the different data sets.
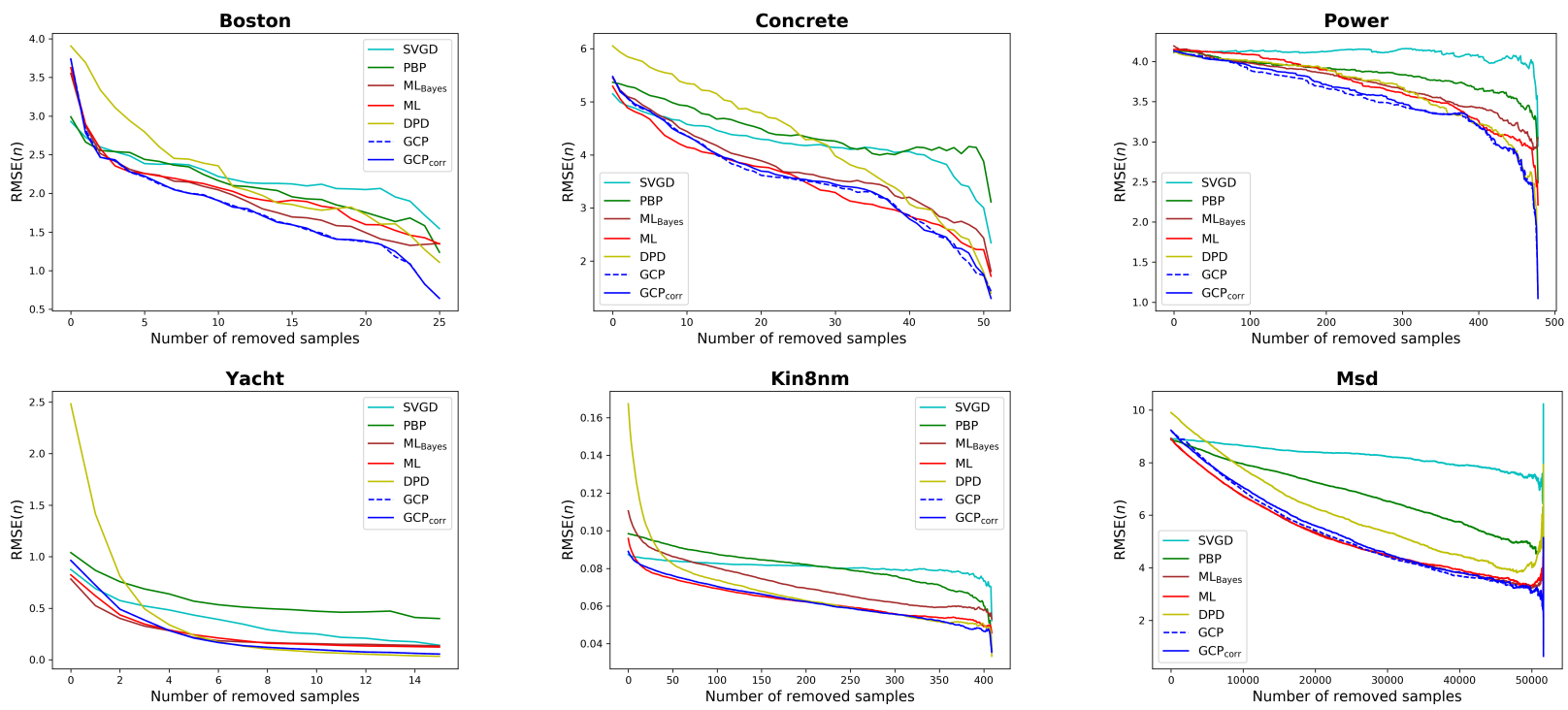

Figure 6.3: The curves $\operatorname{RMSE}(n)$ for the different methods and data sets from Table 6.1 .

feature), and Year Prediction MSD [27] (515345 samples, 90 features). For each data set, a one-dimensional target variable is predicted. Each data set, except for the year prediction MSD, is randomly split into 50 train-test folds with $95 \%$ of samples in each train subset. All the measure values reported below are the averages of the respective measure values over 50 folds. For the year prediction MSD, we used a single split recommended in 27].

Results. Table 6.1 shows the measure values of the different methods. In each column, we mark a method in bold if it is significantly (due to the two-tailed paired difference test with $\mathrm{p}=0.05$ ) better or indistinguishable from all the other methods. We do not present the $\mathrm{GCP}_{\text {corr }}$ results in this table because, in the absence of outliers, it yielded AUC very close to that of GCP. We see that the GCP achieves the best AUC values on all the data sets (except MSD), which indicates the best trade-off between properly learning the mean and the variance.

Figure 6.3 shows the curves $\operatorname{RMSE}(n)$ for the different methods and data sets from Table 6.1. We see that the curve $\operatorname{RMSE}(n)$ typically decays faster for the GCP compared with the other methods. In the absence of outliers, the curves of the GCP and GCP ${ }_{\text {corr }}$ practically coincide, yielding very close AUC values. 


\begin{tabular}{|c|c|c|c|c|c|c|c|c|c|c|c|c|}
\hline \multicolumn{3}{|c|}{ Boston } & \multicolumn{2}{|l|}{ Concrete } & \multicolumn{2}{|l|}{ Power } & \multicolumn{2}{|l|}{ Yacht } & \multicolumn{2}{|l|}{ Kin8nm } & \multicolumn{2}{|l|}{ MSD } \\
\hline & RMSE & AUC & RMSE & AUC & RMSE & AUC & RMSE & AUC & RMSE & AUC & RMSE & AUC \\
\hline SVGD & $11.39 \pm 4.46$ & $7.39 \pm 1.99$ & $16.92 \pm 1.10$ & $16.05 \pm 2.78$ & $5.53 \pm 0.42$ & $5.18 \pm 0.45$ & $13.37 \pm 5.48$ & $6.64 \pm 2.25$ & $0.22 \pm 0.03$ & $0.17 \pm 0.02$ & $9.56 \pm \mathrm{NA}$ & $9.00 \pm \mathrm{NA}$ \\
\hline PBP & $8.64 \pm 2.70$ & $5.70 \pm 1.40$ & $9.88 \pm 1.99$ & $7.62 \pm 1.23$ & $4.60 \pm 0.32$ & $4.27 \pm 0.29$ & $17.55 \pm 29.10$ & $8.40 \pm 4.66$ & $0.15 \pm 0.01$ & $0.13 \pm 0.01$ & $9.05 \pm \mathrm{NA}$ & $8.46 \pm \mathrm{NA}$ \\
\hline$M L_{\text {Bayes }}$ & $4.17 \pm 1.28$ & $3.56 \pm 1.14$ & $6.94 \pm 0.84$ & $6.17 \pm 0.93$ & $4.67 \pm 0.32$ & $4.56 \pm 0.43$ & $2.62 \pm 1.63$ & $1.35 \pm 0.53$ & $0.13 \pm 0.01$ & $0.12 \pm 0.01$ & $9.17 \pm \mathrm{NA}$ & $8.32 \pm \mathrm{NA}$ \\
\hline ML & $3.66 \pm 1.01$ & $3.40 \pm 1.50$ & $6.06 \pm 0.70$ & $5.55 \pm 0.99$ & $4.73 \pm 0.28$ & $4.69 \pm 0.44$ & $2.52 \pm 1.61$ & $1.50 \pm 0.98$ & $0.14 \pm 0.01$ & $0.13 \pm 0.01$ & $9.10 \pm \mathrm{NA}$ & $8.37 \pm \mathrm{NA}$ \\
\hline DPD & $3.74 \pm 1.70$ & $2.21 \pm 0.57$ & $5.17 \pm 0.81$ & $3.80 \pm 0.64$ & $4.13 \pm 0.33$ & $3.82 \pm 0.50$ & $1.37 \pm 0.68$ & $0.27 \pm 0.13$ & $0.15 \pm 0.02$ & $0.07 \pm 0.01$ & $9.80 \pm \mathrm{NA}$ & $6.10 \pm \mathrm{NA}$ \\
\hline GCP & $3.66 \pm 1.54$ & $2.83 \pm 1.36$ & $5.54 \pm 0.68$ & $5.14 \pm 0.94$ & $4.16 \pm 0.31$ & $3.65 \pm 0.37$ & $1.09 \pm 0.56$ & $0.47 \pm 0.33$ & $0.10 \pm 0.01$ & $0.08 \pm 0.01$ & $9.31 \pm \mathrm{NA}$ & $8.16 \pm \mathrm{NA}$ \\
\hline $\mathrm{GCP}_{\text {corr }}$ & $3.66 \pm 1.54$ & $1.97 \pm 0.50$ & $5.54 \pm 0.68$ & $3.65 \pm 0.69$ & $4.16 \pm 0.31$ & $3.64 \pm 0.38$ & $1.09 \pm 0.56$ & $0.29 \pm 0.14$ & $0.10 \pm 0.01$ & $0.07 \pm 0.00$ & $9.31 \pm \mathrm{NA}$ & $5.57 \pm \mathrm{NA}$ \\
\hline
\end{tabular}

Table 6.2: RMSE and AUC for the different data sets with $5 \%$ of outliers.

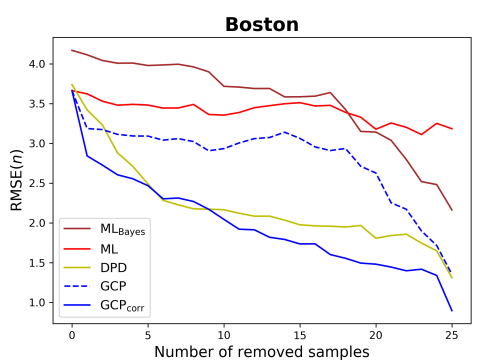

Yacht

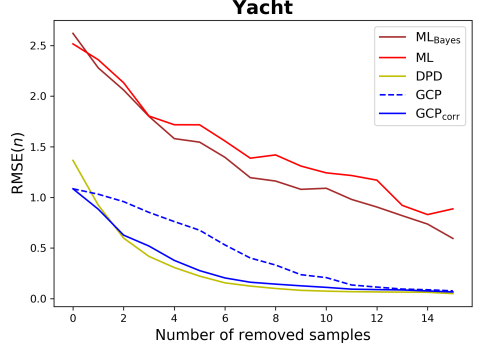

Concrete

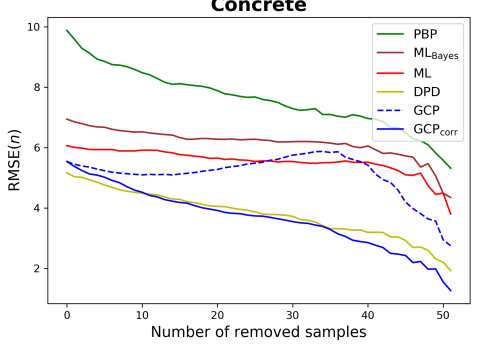

Kin8nm

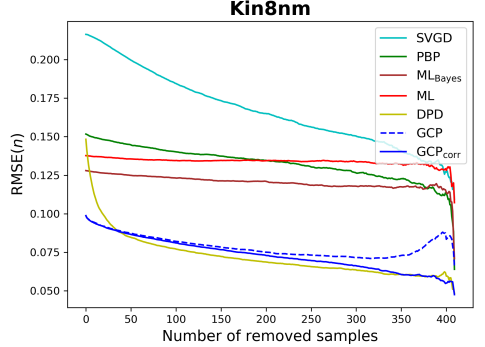

Power

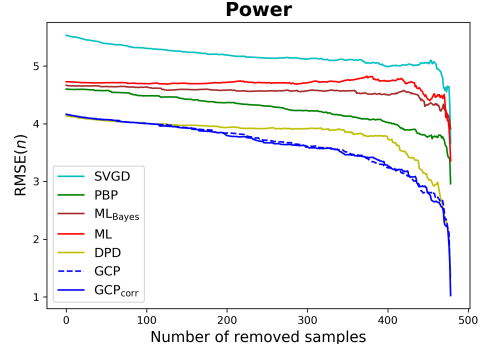

Msd

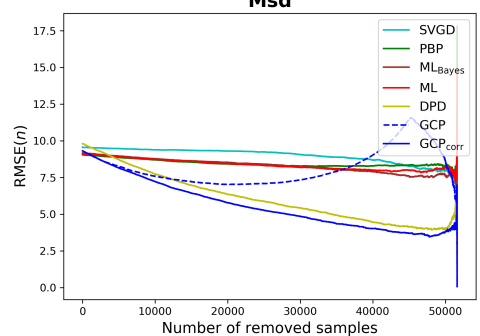

Figure 6.4: The curves $\operatorname{RMSE}(n)$ for the different methods and data sets with $5 \%$ of outliers from Table 6.2. The curves for the PBP and SVGD are not shown on some of the plots because of their high RMSE and AUC values.

\subsection{Real world data sets: outliers in the training data sets}

We analyze the same methods and data sets as in Sec. 6.4, but now contaminated by outliers. For each training set, we randomly choose $5 \%$ of samples and replace them by outliers. The outliers are sampled from the Gaussian distribution with the mean equal to the mean over all the targets in the original training set and standard deviation equal to ten times the standard deviation over the targets in the original training set. The results are presented in Table 6.2. Using the correction formula for $\tilde{V}_{\text {est }}$ in $(2.18)$ allows the $\mathrm{GCP}_{\text {corr }}$ to obtain the best AUC values on all data sets. We also note that the Bayesian methods SVGD and PBP are especially sensitive to outliers, which is reflected in their high RMSE and AUC values.

Figure 6.4 shows the curves $\operatorname{RMSE}(n)$ for the different methods and data sets from Table 6.2. The curves of the $\mathrm{GCP}_{\text {corr }}$ are typically significantly below the corresponding curves of other methods, including the GCP without correction. 


\section{Conclusion}

Our goal was to approximate ground truth probability distributions by parametrically defined distributions. For their unknown parameters, we introduced a prior distribution, whose parameters are learned by neural networks with deterministic weights. In such a setting, one cannot directly update the prior's parameters by the Bayesian rule, but one should rather update the network's weights. Hence, we proposed to replace a full Bayesian update of prior's parameters by a gradient descent step in the direction of minimizing the KL divergence from the posterior to the prior distribution, which we called the GCP update. We showed that the GCP update is equivalent to the gradient ascent step that maximizes the likelihood of the predictive distribution. Interestingly, this result holds in general, independently of whether the posterior and prior distributions belong to the same family or not.

Next, we concentrated on the case where the ground truth distribution is normal with unknown mean and variance. A natural choice for the prior is the normal-gamma distribution. We obtained a dynamical system for its parameters that approximates the corresponding GCP update and analyzed it in detail. It revealed the convergence of the prior's parameters which is quite different from that for the standard Bayesian update, although in both cases the predictive Student's t-distribution converges to the ground truth normal distribution.

Furthermore, we analyzed how the GCP interacts with outliers in the training set. In the presence of outliers, the prior's parameter $\alpha$ (half the number of degrees of freedom of the predictive Student's t-distribution) does not tend to infinity any more. On one hand, this allows for a much better estimate of the mean of the ground truth normal distribution, compared with the ML method. On the other hand, this leads to overestimation of the variance of the ground truth distribution. We obtained, for the first time, an explicit formula that allows one to correct the estimate of the variance and recover the ground truth variance of the normal distribution.

Finally, we validated the GCP neural network on synthetic and six real-world data sets and compared it with the ML, DPD, SVGD, and PBP neural networks. We analyzed both clean data sets and data sets contaminated by $5 \%$ of outliers. We measured the trade-off between properly learning the mean and the variance (reflected in the AUC values) and the overall error (RMSE). The GCP was the only method that demonstrated the best AUC values simultaneously for clean and contaminated data sets.

To conclude, we indicate several directions of future research:

1. In case where the ground truth distribution of $\mathbf{y}$ is multivariate Gaussian with unknown mean and precision matrix, the conjugate prior is given by a family of normal-Wishart distributions. Due to Lemma 2.1 and Remark2.1 (item 3), the gradient conjugate prior update is equivalent to maximizing the likelihood of the predictive distribution, namely, multivariate Student distribution. Hence, one can obtain an analog of system (3.8), which will be a gradient system, too, and whose dynamics will be robust against outliers. Its analysis should allow one to derive a correction formula generalizing 2.4 and to reconstruct the ground truth multivariate Gaussian distribution.

2. A rigorous mathematical analysis of the influence of outliers on the dynamics of the 
prior's parameters seems to be feasible. One can relate the percentage of the outliers and a type of distribution they come from with the dynamical system (3.8), in which the expectations will be taken with respect to the new distribution (mixture of normal and the one from which the outliers are sampled). Further comparison with Bayesian methods based on robust divergences [6] and generalizing the DPD method is also needed.

3. Section 4 shows that one can fix $\alpha$ and still recover the ground truth normal distribution, while Sec. 5 indicates how different values of $\alpha$ may influence the learning speed in clean and noisy regions. The influence of $\alpha$ on the fit of the GCP neural networks for real-world data sets would be an interesting practical question. Our preliminary analysis showed that fixing large $\alpha$ was beneficial for the largest MSD data set. For example, fixing $\alpha=30$ yielded $\mathrm{RMSE}=8.89$ and $\mathrm{AUC}=5.13$ (cf. Table 6.1).

4. It is worth checking the GCP networks for other choices of ground truth and prior distributions.

5. The use of ensembles of MLs (called deep ensembles) was recently proposed in 23 , 24]. It is worth studying ensembles of GCPs capturing both aleatoric and epistemic uncertainty and being robust against outliers. Another possibility to capture epistemic uncertainty is to treat weights of the networks as random variables and learn their posterior distribution as is usually done for Bayesian neural networks.

\section{A Properties of the function $A(\alpha)$ : proof of Lemma 3.1}

For $\alpha>0$ and $A \in[0, \alpha]$, we study equation (3.15), which is equivalent to the following:

$$
E(\alpha, A):=\frac{1}{(2 \pi)^{1 / 2}} \int_{-\infty}^{\infty} \frac{(2 \alpha+1) z^{2}}{2(\alpha-A)+z^{2}} e^{-\frac{z^{2}}{2}} d z-1=0 .
$$

Lemma A.1. For each $\alpha>0$, equation A.1) has a unique root $A=A(\alpha) \in(0, \alpha)$. Furthermore,

$$
A(\alpha)=\alpha-\frac{4}{\pi} \alpha^{2}+o\left(\alpha^{2}\right) \text { as } \alpha \rightarrow 0
$$

Proof. 1. Note that $E(\alpha, A)$ is increasing with respect to $A \in(0, \alpha)$ and $E(\alpha, \alpha)=2 \alpha>0$. Hence, it remains to show that $E(\alpha, 0)<0$. We have

$$
E(\alpha, 0)=\frac{1}{(2 \pi)^{1 / 2}} \int_{-\infty}^{\infty} \frac{(2 \alpha+1) z^{2}}{2 \alpha+z^{2}} e^{-\frac{z^{2}}{2}} d z-1=\frac{1}{(2 \pi)^{1 / 2}} \int_{-\infty}^{\infty} \frac{z^{2}}{2 \alpha+z^{2}}\left(1-z^{2}\right) e^{-\frac{z^{2}}{2}} d z
$$

Now the inequality $E(\alpha, 0)<0$ follows from $A .2$ and the monotonicity of $\frac{z^{2}}{2 \alpha+z^{2}}$. Indeed,

$$
\begin{aligned}
\int_{0}^{1} \frac{z^{2}}{2 \alpha+z^{2}}\left(1-z^{2}\right) e^{-\frac{z^{2}}{2}} d z & <\int_{0}^{1} \frac{1}{2 \alpha+1}\left(1-z^{2}\right) e^{-\frac{z^{2}}{2}} d z \\
& =\int_{1}^{\infty} \frac{1}{2 \alpha+1}\left(z^{2}-1\right) e^{-\frac{z^{2}}{2}} d z<\int_{1}^{\infty} \frac{z^{2}}{2 \alpha+z^{2}}\left(z^{2}-1\right) e^{-\frac{z^{2}}{2}} d z
\end{aligned}
$$


where we have used the equality

$$
\int_{0}^{\infty} e^{-\frac{z^{2}}{2}} d z=\int_{0}^{\infty} z^{2} e^{-\frac{z^{2}}{2}} d z=\left(\frac{\pi}{2}\right)^{1 / 2} .
$$

2. Now we prove the asymptotics of $A(\alpha)$. Using the function $F(x)$ defined in $(3.16)$, we rewrite equation A.1 in the form

$$
(2 \alpha+1)(\alpha-A) F(\alpha-A)-\alpha=0 .
$$

Using the expansion of $\operatorname{erfc}(x)$ around 0 (see [1, Sec. 7.1.6]) and formula (3.16), we have for all $x>0$

$$
x F(x)=\frac{\pi^{1 / 2}+\delta}{2} x^{1 / 2}, \quad \delta=o(1) \text { as } x \rightarrow 0 .
$$

Now, for each $\alpha>0$, we represent $A=\alpha-k^{2} \alpha^{2}$ and prove that $k=\frac{2}{\pi^{1 / 2}}+o(1)$ as $\alpha \rightarrow 0$. Combining the representation of $A$ with A.3 and A.4 , we obtain

$$
\left(-1+\frac{k \pi^{1 / 2}}{2}+\frac{k \delta}{2}\right)+k\left(\delta+\pi^{1 / 2}\right) \alpha=0 .
$$

Obviously, if $\alpha=\delta=0$, we have $k=\frac{2}{\pi^{1 / 2}}$. Hence, by the implicit function theorem, $k=\frac{2}{\pi^{1 / 2}}+o(1)$ as $\alpha, \delta \rightarrow 0$. Recalling that $\delta=o(1)$ as $\alpha \rightarrow 0$, we complete the proof.

Lemma A.2. For each $\alpha \geq 1$, equation A.1 has a unique root $A=A(\alpha) \in(0,1)$. Furthermore,

$$
A(\alpha)=1-\frac{3}{2 \alpha}+o\left(\frac{1}{\alpha}\right) \text { as } \alpha \rightarrow \infty .
$$

Proof. 1. In the proof of Lemma A.1, we have shown that $E(\alpha, 0)<0$. Due to the monotonicity of $E(\alpha, A)$ with respect to $A \in(0, \alpha)$, it remains to show that $E(\alpha, 1)>0$. Using that $2 \alpha-2 \geq 0$, we have

$$
\begin{aligned}
E(\alpha, 1) & =\frac{1}{(2 \pi)^{1 / 2}} \int_{-\infty}^{\infty} \frac{(2 \alpha+1) z^{2}}{2 \alpha-2+z^{2}} e^{-\frac{z^{2}}{2}} d z-1=\frac{1}{(2 \pi)^{1 / 2}} \int_{-\infty}^{\infty} \frac{z^{2}\left(3-z^{2}\right)}{2 \alpha-2+z^{2}} e^{-\frac{z^{2}}{2}} d z \\
& =\frac{1}{(2 \pi)^{1 / 2}} \int_{-\infty}^{\infty} \int_{0}^{\infty} z^{2}\left(3-z^{2}\right) e^{-z^{2} / 2-\left(2 \alpha-2+z^{2}\right) \xi} d \xi d z \\
& =\frac{1}{(2 \pi)^{1 / 2}} \int_{0}^{\infty} e^{-(2 \alpha-2) \xi} d \xi \int_{-\infty}^{\infty} z^{2}\left(3-z^{2}\right) e^{-(\xi+1 / 2) z^{2}} d z \\
& =3 \int_{0}^{\infty} \exp (-(2 \alpha-2) \xi)\left(\frac{1}{(2 \xi+1)^{3 / 2}}-\frac{1}{(2 \xi+1)^{5 / 2}}\right) d \xi>0 .
\end{aligned}
$$

2. Now we prove the asymptotics of $A(\alpha)$. Using the expansion of $\operatorname{erfc}(x)$ around $\infty$ (see [1, Sec. 7.1.23]) and formula (3.16), we have for all $x>0$

$$
x F(x)=\frac{1}{2}-\frac{1}{4 x}+\frac{3}{8 x^{2}}-\frac{15+\delta}{16 x^{3}}, \quad \delta=o(1) \text { as } x \rightarrow \infty .
$$


Now, for each $\alpha>0$, we representing $A=1-k / \alpha$ and prove that $k=3 / 2+o(1)$ as $\alpha \rightarrow \infty$. Combining the representation of $A$ with A.3 and (A.5), we obtain

$$
\frac{-12+38 k z^{2}-28 k^{2} z^{3}+8 k^{3} z^{4}+8 k-28 k z-33 z-2 \delta+16 k^{2} z^{2}-z \delta}{\left(1-z+k z^{2}\right)^{3}}=0, \quad z:=\frac{1}{\alpha} .
$$

Obviously, if $\alpha=\delta=0$, we have $k=3 / 2$. Hence, by the implicit function theorem, $k=3 / 2+o(1)$ as $z, \delta \rightarrow 0$. Recalling that $\delta=o(1)$ as $z \rightarrow 0$, we complete the proof.

Lemma A.3. The function $A(\alpha)$ satisfies the differential equation in (3.17).

Proof. Denoting by $E_{\alpha}$ and $E_{A}$ the partial derivatives of the function $E(\alpha, A)$ with respect to $\alpha$ and $A$, respectively, and using the implicit function theorem, we have

$$
A^{\prime}=-E_{A}^{-1} E_{\alpha}
$$

Since $A(\alpha)$ satisfies the equation in A.1 , we obtain

$$
\begin{aligned}
E_{\alpha} & =-2 \frac{1}{(2 \pi)^{1 / 2}} \int_{-\infty}^{\infty} \frac{(2 \alpha+1) z^{2}}{\left(2(\alpha-A)+z^{2}\right)^{2}} e^{-z^{2} / 2} d z+\frac{1}{(2 \pi)^{1 / 2}} \int_{-\infty}^{\infty} \frac{2 z^{2}}{\left(2(\alpha-A)+z^{2}\right)} e^{-z^{2} / 2} d z \\
& =-E_{A}+\frac{2}{2 \alpha+1}
\end{aligned}
$$

Now we calculate $E_{A}$ and integrate by parts:

$$
E_{A}=\frac{1}{(2 \pi)^{1 / 2}} \int_{-\infty}^{\infty} \frac{(2 \alpha+1)(2 z)}{\left(2(\alpha-A)+z^{2}\right)^{2}} z e^{-z^{2} / 2} d z=\frac{1}{(2 \pi)^{1 / 2}} \int_{-\infty}^{\infty} \frac{(2 \alpha+1)\left(1-z^{2}\right)}{\left(2(\alpha-A)+z^{2}\right)} e^{-z^{2} / 2} d z
$$

Again using the equality in (A.1), we obtain

$$
\begin{aligned}
E_{A} & =\frac{1}{(2 \pi)^{1 / 2}} \int_{-\infty}^{\infty} \frac{2 \alpha+1}{\left(2(\alpha-A)+z^{2}\right)} e^{-z^{2} / 2} d z-1 \\
& =\frac{1}{2(\alpha-A)} \frac{1}{(2 \pi)^{1 / 2}} \int_{-\infty}^{\infty} \frac{(2 \alpha+1)\left(2(\alpha-A)+z^{2}-z^{2}\right)}{\left(2(\alpha-A)+z^{2}\right)} e^{-z^{2} / 2} d z-1 \\
& =\frac{2 \alpha+1-1}{2(\alpha-A)}-1=\frac{\alpha}{\alpha-A}-1 .
\end{aligned}
$$

Combining A.6 - A.8, we obtain (3.17).

Lemma A.4. For all $\alpha>0$, we have $A^{\prime}(\alpha)>0$ and

$$
\frac{2 \alpha}{2 \alpha+3}<A(\alpha)
$$


Proof. It suffices to show that the right-hand side of (3.17) is positive for all $\alpha>0$, which is equivalent to A.9. We consider the function

$$
g(\alpha):=A(\alpha)-\frac{2 \alpha}{2 \alpha+3}
$$

and show that $g(\alpha)>0$ for all $\alpha>0$. Assume this is not true. Since $g(\alpha)>0$ for all sufficiently small $\alpha>0$ (due to the asymptotics in Lemma A.1) and $\lim _{\alpha \rightarrow \infty} g(\alpha)=0$ (due to the asymptotics in Lemma A.2, this would imply that

$$
g^{\prime}(\alpha)=0, \quad g(\alpha) \leq 0 \quad \text { for some } \alpha>0 .
$$

Using the fact that $\left(\frac{2 \alpha}{2 \alpha+3}\right)^{\prime}>0$ and applying Lemma A.3, we have

$$
g^{\prime}(\alpha)<A^{\prime}(\alpha)=\frac{2 \alpha(A-1)+3 A}{(2 \alpha+1) A} .
$$

Since $A(\alpha) \leq \frac{2 \alpha}{2 \alpha+3}$ for $\alpha$ in A.10, we obtain

$$
g^{\prime}(\alpha)<\frac{1}{(2 \alpha+1) A}\left(2 \alpha\left(\frac{2 \alpha}{2 \alpha+3}-1\right)+\frac{6 \alpha}{2 \alpha+3}\right)=0
$$

which contradicts A.10.

\section{B Proof of Lemma 3.2}

1. Let us show that $\dot{\nu}=0$ if $\sigma=\sigma_{0}(\alpha)$. Due to 3.14,

$$
\left.\mathbb{E}\left[\frac{\partial K}{\partial \nu}\right]\right|_{\sigma=\sigma_{0}(\alpha)}=\frac{1}{2 \nu(\nu+1)}\left(\frac{1}{(2 \pi)^{1 / 2}} \int_{-\infty}^{\infty} \frac{(2 \alpha+1) z^{2}}{2(\alpha-A)+z^{2}} e^{-\frac{z^{2}}{2}} d z-1\right) .
$$

Now the equation $\left.\mathbb{E}\left[\frac{\partial K}{\partial \nu}\right]\right|_{\sigma=\sigma_{0}(\alpha)}=0$ can be rewritten as follows:

$$
\int_{-\infty}^{\infty} \frac{(2 \alpha+1) z^{2}}{2(\alpha-A)+z^{2}} e^{-\frac{z^{2}}{2}} d z-1=0
$$

which is equivalent to 3.15). By Lemma 3.1, it has a unique root $A=A(\alpha) \in(0, \min (\alpha, 1))$.

Since $\mathbb{E}\left[\frac{\partial K}{\partial \nu}\right]$ is decreasing with respect to $\sigma$ due to 3.14 , the assertions about $\nu$ in 3.25 follow.

2. Next, we show that $\dot{\beta}=0$ if $\sigma=\sigma_{0}(\alpha)$. Due to 3.13$), 3.15$, and (3.16),

$$
\begin{aligned}
\left.\mathbb{E}\left[\frac{\partial K}{\partial \beta}\right]\right|_{\sigma=\sigma_{0}(\alpha)} & =\frac{\nu+1}{\nu V}\left(\frac{1}{(2 \pi)^{1 / 2}} \int_{-\infty}^{\infty} \frac{2 \alpha+1}{2(\alpha-A)+z^{2}} e^{-\frac{z^{2}}{2}} d z-\frac{\alpha}{\alpha-A}\right) \\
& =\frac{\nu+1}{\nu V}\left((2 \alpha+1) F(\alpha-A)-\frac{\alpha}{\alpha-A}\right)=0 .
\end{aligned}
$$

Since the expression in the brackets in 3.13 is increasing with respect to $\sigma$, the assertions about $\beta$ in 3.25 follow. 


\section{Proof of Lemma 3.3}

Using the equality $\frac{1}{(2 \pi)^{1 / 2}} \int_{-\infty}^{\infty} e^{-z^{2} / 2} d z=1$, we rewrite (3.14) as follows:

$$
\mathbb{E}\left[\frac{\partial K}{\partial \nu}\right]=-\frac{1}{\nu(\nu+1)}\left(\frac{\alpha+1 / 2}{(2 \pi)^{1 / 2}} \int_{-\infty}^{\infty} \frac{1}{1+\frac{V}{\sigma} \frac{z^{2}}{2}} e^{-\frac{z^{2}}{2}} d z-\alpha\right) .
$$

Combining this relation with (3.13) shows that the points $(\nu(t), \beta(t))$ belong to the integral curves of the differential equation $(3.22)$, because

$$
\frac{d \beta}{d \nu}=\frac{\mathbb{E}\left[\frac{\partial K}{\partial \beta}\right]}{\mathbb{E}\left[\frac{\partial K}{\partial \nu}\right]}=-\frac{\nu(\nu+1)}{\beta}
$$

Separating variables in this equation, one can see that the integral curves are given by (3.21).

\section{Proof of Lemma 3.4}

1. Due to 3.12 ,

$$
\begin{aligned}
\left.\mathbb{E}\left[\frac{\partial K}{\partial \alpha}\right]\right|_{\sigma=\sigma_{\varkappa}(\alpha)} & =\frac{1}{(2 \pi)^{1 / 2}} \int_{-\infty}^{\infty} \ln \left(1+\frac{1}{\left(1-\frac{\varkappa}{\alpha^{2}}\right)(\alpha-A)} \frac{z^{2}}{2}\right) e^{-\frac{z^{2}}{2}} d z \\
& +\Psi(\alpha)-\Psi\left(\alpha+\frac{1}{2}\right)
\end{aligned}
$$

Using that $\Psi(\alpha)-\Psi(\alpha+1 / 2) \rightarrow 0$ and $A(\alpha) \rightarrow 1$ as $\alpha \rightarrow \infty$, we see that

$$
\left.\lim _{\alpha \rightarrow \infty} \mathbb{E}\left[\frac{\partial K}{\partial \alpha}\right]\right|_{\sigma=\sigma_{\varkappa}(\alpha)}=0
$$

2. To complete the proof of (3.26), it suffices (due to (D.2)) to show that the derivative of the right-hand side in (D.1) is positive for $\varkappa=0$ and all $\alpha>0$. We denote the derivative of the right-hand side in (D.1) by $G_{\varkappa}(\alpha)$. To calculate it, we set

$$
B(\alpha):=1-\frac{\varkappa}{\alpha}, \quad \gamma(\alpha)=\frac{1}{2 B(\alpha-A)} .
$$

Then

$$
\frac{\partial}{\partial \alpha} \ln \left(1+\gamma z^{2}\right)=-\frac{z^{2}}{1+\gamma z^{2}}\left(\frac{\left(1-A^{\prime}\right)}{2 B(\alpha-A)^{2}}+\frac{B^{\prime}}{2 B^{2}(\alpha-A)}\right) .
$$

If $\varkappa=0$, then $B=1, B^{\prime}=0$, and (due to (A.1)

$$
\frac{1}{(2 \pi)^{1 / 2}} \int_{-\infty}^{\infty} \frac{z^{2}}{1+\gamma z^{2}}=\frac{1}{(2 \pi)^{1 / 2}} \int_{-\infty}^{\infty} \frac{z^{2}}{1+\frac{1}{2(\alpha-A)} z^{2}}=\frac{2(\alpha-A)}{2 \alpha+1}
$$


Using (D.3) and (D.4), we obtain

$$
G_{0}(\alpha)=-\frac{1-A^{\prime}}{(2 \alpha+1)(\alpha-A)}+\Psi^{\prime}(\alpha)-\Psi^{\prime}\left(\alpha+\frac{1}{2}\right),
$$

or, using Lemma 3.1 (item 4), equivalently,

$$
G_{0}(\alpha)=-\frac{2}{(2 \alpha+1)^{2} A}+\Psi^{\prime}(\alpha)-\Psi^{\prime}\left(\alpha+\frac{1}{2}\right) .
$$

Due to the inequality $A(\alpha)>\frac{2 \alpha}{2 \alpha+3}$ (see the first inequality in Lemma 3.1, item 3,

$$
G_{0}(\alpha)>\tilde{G}_{0}(\alpha)
$$

where

$$
\tilde{G}_{0}(\alpha)=-\frac{2 \alpha+3}{(2 \alpha+1)^{2} \alpha}+\Psi^{\prime}(\alpha)-\Psi^{\prime}\left(\alpha+\frac{1}{2}\right) .
$$

Therefore, for the proof of 3.26 it remains to show that

$$
\tilde{G}_{0}(\alpha)>0 \text { for all } \alpha>0
$$

2.1. First, we prove (D.6) for large $\alpha$. Using the asymptotics [1, Sec. 6.4.12]

$$
\Psi^{\prime}(\alpha)-\Psi^{\prime}\left(\alpha+\frac{1}{2}\right)=\frac{1}{2 \alpha^{2}}+\frac{1}{4 \alpha^{3}}+O\left(\frac{1}{\alpha^{5}}\right) \quad \text { as } \alpha \rightarrow \infty
$$

we obtain from (D.5)

$$
\tilde{G}_{0}(\alpha)=\frac{3}{8 \alpha^{4}}+O\left(\frac{1}{\alpha^{5}}\right)>0 \text { for all sufficiently large } \alpha \text {. }
$$

2.2. Due to (D.8), to complete the proof of (D.6) it now suffices to show that

$$
\tilde{G}_{0}(\alpha)-\tilde{G}_{0}(\alpha+1)>0 \text { for all } \alpha>0
$$

Applying the recurrence relation $\Psi^{\prime}(z+1)=\Psi^{\prime}(z)-1 / z^{2}$ (see [1, Sec. 6.4.6]), we obtain from $(\mathrm{D} .5)$

$$
\begin{aligned}
\tilde{G}_{0}(\alpha)-\tilde{G}_{0}(\alpha+1) & =-\frac{2 \alpha+3}{(2 \alpha+1)^{2} \alpha}+\frac{2(\alpha+1)+3}{(2(\alpha+1)+1)^{2}(\alpha+1)}+\frac{1}{\alpha^{2}}-\frac{1}{(\alpha+1 / 2)^{2}} \\
& =\frac{3(4 \alpha+3)}{(2 \alpha+1)(\alpha+1)(2 \alpha+3)^{2} \alpha^{2}}>0,
\end{aligned}
$$

which proves (D.6) and thus completes the proof of 3.26 . 
3. Now consider the case $\varkappa>0$. Note that the expression in the brackets in $(\bar{D} .3)$ is positive due to Lemma 3.1 and the fact that $B^{\prime}(\alpha)>0$. Hence, we obtain from (D.3)

$$
\begin{aligned}
\frac{\partial}{\partial \alpha} \ln \left(1+\gamma z^{2}\right) & =-2 B(\alpha-A) \frac{z^{2}}{2 B(\alpha-A)+z^{2}}\left(\frac{1-A^{\prime}}{2 B(\alpha-A)^{2}}+\frac{B^{\prime}}{2 B^{2}(\alpha-A)}\right) \\
& <-\frac{z^{2}}{2(\alpha-A)+z^{2}}\left(\frac{1-A^{\prime}}{\alpha-A}+\frac{B^{\prime}}{B}\right) .
\end{aligned}
$$

Combining the latter inequality with (D.4) and using Lemma 3.1 (item 4), we have

$$
G_{\varkappa}(\alpha)<-\frac{2}{(2 \alpha+1)^{2} A}-\frac{B^{\prime}}{B(2 \alpha+1)}+\Psi^{\prime}(\alpha)-\Psi^{\prime}\left(\alpha+\frac{1}{2}\right) .
$$

Additionally using the asymptotics in (D.7) and the expansion of $A(\alpha)$ in (3.18) as $\alpha \rightarrow \infty$, we obtain

$$
G_{\varkappa}(\alpha)<-\frac{\varkappa}{2 \alpha^{3}}+o\left(\frac{1}{\alpha^{3}}\right)<0 \text { for all sufficiently large } \alpha \text {. }
$$

\section{E Hyperparameters}

When we fit different methods on the real world data sets, we normalize them so that the input features and the targets have zero mean and unit variance in the training set. We used minibatch 5 on Boston, Concrete, and Yacht, minibatch 10 on Power and Kin8nm, and minibatch 5000 on MSD. We used Adam (with $\beta_{1}=0.9, \beta_{2}=0.999$ ), RmsProp (with $\rho=0.5$ ), or Nesterov momentum (with momentum 0.9) optimizers for fitting the ML, DPD, and GCP methods. In case of each optimizer, we performed a grid search for the learning rate in the range $\{0.00002,0.00005,0.0001,0.0002,0.0007,0.001,0.005\}$ and for the dropout rate in the range $\{0,0.1,0.2,0.3,0.4\}$. The optimizers and the parameters yielding the best AUC are presented in Tables E.1 and E.2. We trained the SVGD, using the authors cod $\$^{5}$, for 3000 epochs with the learning rate chosen by the grid search in the range $\{0.00005,0.0001,0.0005,0.001,0.005,0.001\}$. The optimal learning rate was 0.001 for Boston, 0.005 for Concrete, 0.005 for Power, 0.001 for Yacht, 0.005 for Kin8nm, and 0.005 for MSD. We trained the PBP, using the authors' cod $\AA^{6}$ for 40 epochs as recommended in [13] (the learning rate need not be fine tuned because it is absent in the PBP as such). For the $\mathrm{ML}_{\text {drop }}$, we used the same hyperparameters as for the ML, except for the dropout rate, which was set to 0.4 and used for both training and prediction. For prediction, we used 50 samples as suggested in [19].

Acknowledgements. Both authors would like to thank the DFG project SFB 910. The research of the first author was also supported by the DFG Heisenberg Programme and by the "RUDN University Program 5-100". The authors are grateful to anonymous referees for their comments and suggestions, which significantly clarified and improved our presentation.

\footnotetext{
${ }^{5}$ https://github.com/DartML/Stein-Variational-Gradient-Descent

${ }^{6}$ https://github.com/HIPS/Probabilistic-Backpropagation
} 


\begin{tabular}{|c|c|c|c|c|c|c|c|c|c|c|c|c|}
\hline \multicolumn{5}{|c|}{ Boston } & \multicolumn{4}{|l|}{ Concrete } & \multicolumn{4}{|l|}{ Power } \\
\hline & Optimizer & $\begin{array}{c}\text { Learning } \\
\text { rate }\end{array}$ & Dropout & $\begin{array}{c}\text { Number } \\
\text { of epochs }\end{array}$ & Optimizer & $\begin{array}{c}\text { Learning } \\
\text { rate }\end{array}$ & Dropout & $\begin{array}{c}\text { Number } \\
\text { of epochs }\end{array}$ & Optimizer & $\begin{array}{c}\text { Learning } \\
\text { rate }\end{array}$ & Dropout & $\begin{array}{c}\text { Number } \\
\text { of epochs }\end{array}$ \\
\hline ML & Adam & 0.0001 & 0.4 & 700 & Adam & 0.0001 & 0.1 & 800 & Adam & 0.00005 & 0 & 150 \\
\hline $\mathrm{ML}_{\text {Bayes }}$ & Adam & 0.0001 & 0.4 & 700 & Adam & 0.0001 & 0.1 & 800 & Adam & 0.00005 & 0.2 & 150 \\
\hline DPD & Nesterov & 0.00002 & 0.4 & 5000 & Nesterov & 0.00001 & 0.1 & 5000 & Adam & 0.0001 & 0 & 400 \\
\hline GCP & Adam & 0.0001 & 0.3 & 700 & Adam & 0.0001 & 0.1 & 1000 & Adam & 0.00005 & 0 & 150 \\
\hline
\end{tabular}

Table E.1: Optimizers and hyperparameters for the ML, DPD, and GCP methods for the Boston, Concrete, and Power data sets.

\begin{tabular}{|c|c|c|c|c|c|c|c|c|c|c|c|c|}
\hline \multicolumn{5}{|c|}{ Yacht } & \multicolumn{4}{|l|}{ Kin8nm } & \multicolumn{4}{|l|}{ MSD } \\
\hline & Optimizer & $\begin{array}{l}\text { Learning } \\
\text { rate }\end{array}$ & Dropout & $\begin{array}{c}\text { Number } \\
\text { of epochs }\end{array}$ & Optimizer & $\begin{array}{l}\text { Learning } \\
\text { rate }\end{array}$ & Dropout & $\begin{array}{l}\text { Number } \\
\text { of epochs }\end{array}$ & Optimizer & $\begin{array}{l}\text { Learning } \\
\text { rate }\end{array}$ & Dropout & $\begin{array}{l}\text { Number } \\
\text { of epochs }\end{array}$ \\
\hline ML & Adam & 0.0001 & 0.1 & 2000 & Adam & 0.0002 & 0 & 200 & Adam & 0.005 & 0.1 & 150 \\
\hline $\mathrm{ML}_{\text {Bayes }}$ & Adam & 0.0001 & 0.1 & 4000 & Adam & 0.0002 & 0.1 & 200 & Adam & 0.005 & 0.1 & 150 \\
\hline DPD & Adam & 0.0002 & 0.1 & 2500 & Adam & 0.0001 & 0 & 400 & Adam & 0.005 & 0.1 & 100 \\
\hline GCP & RmsProp & 0.001 & 0.1 & 1000 & Nesterov & 0.0007 & 0 & 250 & Adam & 0.001 & 0.1 & 200 \\
\hline
\end{tabular}

Table E.2: Optimizers and hyperparameters for the ML, DPD, and GCP methods for the Yacht, Kin8nm, and MSD data sets.

\section{References}

[1] M. Abramowitz, I. Stegun. Handbook of Mathematical Functions with Formulas, Graphs, and Mathematical Tables. National Bureau of Standards Applied Mathematics Series, 55, 1965.

[2] A. Basu, I.R. Harris, N.L. Hjort, M.C. Jones. Robust and efficient estimation by minimising a density power divergence. Biometrika, 85 (1998), 549-559.

[3] C. Bishop. Pattern Recognition and Machine Learning. Springer, 2006

[4] C. Blundell, J. Cornebise, K. Kavukcuoglu and D. Wierstra, Weight uncertainty in neural networks, In Proceedings of the 32nd International Conference on Machine Learning, 7-9 July 2015, Lille, France, JMLR: W\&CP 37, 1613 (2016)

[5] S. Depeweg, J.M. Hernánandez-Lobato,F. Doshi-Velez, S. Udluft, Decomposition of uncertainty in Bayesian deep learning for efficient and risk-sensitive learning, Proceedings of the 35th International Conference on Machine Learning, Stockholm, Sweden, PMLR $80,2018$.

[6] F. Futami, I. Sato and M. Sugiyama: Variational inference based on robust divergences, 31st Annual Conference on Neural Information Processing Systems (NIPS 2017), 4-9 December 2017, Long Beach, CA, USA (2017)

[7] Y. Gal. Uncertainty in Deep Learning. PhD thesis, University of Cambridge, 2016.

[8] Y. Gal and Z. Ghahramani, Dropout as a bayesian approximation: Representing model uncertainty in deep learning, In Proceedings of the 33rd International Conference on Machine Learning, 20-22 June 2016, New York, New York, USA, JMLR: W\&CP 48, 1050 (2016) 
[9] J. Gerritsma, R. Onnink, and A. Versluis. Geometry, resistance and stability of the delft systematic yacht hull series. In International Shipbuilding Progress, 28 (1981), 276-297.

[10] A. Ghosh and A. Basu: Robust Bayes estimation using the density power divergence, Annals of the Institute of Statistical Mathematics, 68(2), 413 (2016)

[11] P. Gurevich, H. Stuke. Learning uncertainty in regression tasks by deep neural networks. arXiv:1707.07287 [stat.ML] (2017).

[12] D. Harrison, D. L. Rubinfeld. Hedonic prices and the demand for clean air, J. Environ. Economics and Management, 5 (1978), 81-102.

[13] J. M. Hernández-Lobato, R. P. Adams. Probabilistic backpropagation for scalable learning of bayesian neural networks. arXiv:1502.05336 [stat.ML] (2015).

[14] J. M. Hernández-Lobato, Y. Li, M. Rowland, T. Bui, D. Hernández-Lobato and R. Turner, Black-box alpha divergence minimization, In Proceedings of the 33rd International Conference on Machine Learning, 20-22 June 2016, New York, New York, USA, JMLR: W\&CP 48, 1511 (2016)

[15] G. Hinton, D. V. Camp. Keeping neural networks simple by minimizing the description length of the weights. In Proceedings of the Sixth Annual Conference on Computational Learning Theory (1993), 5-13.

[16] J. T. G. Hwang, A. A. Ding, Prediction intervals for artificial neural networks, J. Amer. Stat. Assoc, 92, No. 438 (1997), 748-757.

[17] P. Jylänki, A. Nummenmaa, A. Vehtari. Expectation propagation for neural networks with sparsity-promoting priors. The Journal of Machine Learning Research, 15 (2014), 1849-1901.

[18] H. Kaya, P. Tüfekci , S. F. Gürgen: Local and global learning methods for predicting power of a combined gas and steam turbine, Proceedings of the International Conference on Emerging Trends in Computer and Electronics Engineering ICETCEE (2012), 13-18.

[19] A. Kendall, Y. Gal. What uncertainties do we need in Bayesian deep learning for computer Vision?, 31st Conference on Neural Information Processing Systems (NIPS 2017).

[20] A. Khosravi, S. Nahavandi, D. Creighton, A. Atiya, Comprehensive review of neural network-based prediction intervals and new advances, IEEE Trans. Neural Networks, 22, No. 9 (2011), 1341-1356.

[21] D. P. Kingma, T. Salimans and M. Welling, Variational Dropout and the Local Reparameterization Trick, 29th Conference on Neural Information Processing Systems (NIPS 2015), 7-12 December 2015, Palais des Congrès de Montréal, Montréal, Canada, Advances in Neural Information Processing Systems 28, 2575 (2015) 
[22] D. Kingma, and M. Welling, Auto-encoding variational Bayes. ICLR, 2014.

[23] B. Lakshminarayanan, A. Pritzel, C. Blundell. Simple and scalable predictive uncertainty estimation using deep ensembles. Workshop on Bayesian Deep Learning, NIPS 2016, Barcelona, Spain.

[24] B. Lakshminarayanan, A. Pritzel, C. Blundell. Simple and scalable predictive uncertainty estimation using deep ensembles. 31st Conference on Neural Information Processing Systems (NIPS 2017), Long Beach, CA, USA.

[25] Y. Li and Y. Gal, Dropout inference in Bayesian neural networks with alpha-divergences, In Proceedings of the 34th International Conference on Machine Learning, 6-11 August 2017, International Convention Centre, Sydney, Australia, PMLR 70, 2052 (2017)

[26] Y. Li and R. Turner, Rényi divergence variational inference, 30th Conference on Neural Information Processing Systems (NIPS 2016), 5-10 December 2016, Centre Convencions Internacional Barcelona, Barcelona, Spain, Advances in Neural Information Processing Systems 29 (2016)

[27] M. Lichman. UCI Machine Learning Repository [http://archive.ics.uci.edu/ml]. Irvine, CA: University of California, School of Information and Computer Science (2013).

[28] Q. Liu, D. Wang, Stein variational gradient descent: a general purpose Bayesian inference algorithm. 30th Conference on Neural Information Processing Systems (NIPS 2016), Barcelona, Spain (2016).

[29] C. Louizos and M. Welling, Multiplicative normalizing flows for variational Bayesian neural networks, In Proceedings of the 34th International Conference on Machine Learning, 6-11 August 2017, International Convention Centre, Sydney, Australia, PMLR 70, $2218(2017)$

[30] A. Lucas. Outlier Robust Unit Root Analysis. PhD Thesis. 1996.

[31] D. MacKay. A practical Bayesian framework for backpropagation networks. Neural computation, 4 (1992), 448-472.

[32] T. Minka. A family of algorithms for approximate Bayesian inference. PhD thesis, Massachusetts Institute of Technology, 2001.

[33] K. Murphy. Machine Learning. A Probabilistic Perspective. MIT Press. Cambridge, 2012.

[34] P. Myshkov, S. Julier. Posterior distribution analysis for Bayesian inference in neural networks. Workshop on Bayesian Deep Learning, NIPS 2016, Barcelona, Spain.

[35] S. Nadarajah, S. Kotz. Estimation methods for the multivariate t-distribution. Acta Appl Math 102 (2008), 99-118. 
[36] R. Neal. Bayesian learning for neural networks. PhD thesis, University of Toronto, 1995.

[37] D. A. Nix and A. S. Weigend, Estimating the mean and variance of the target probability distribution, in Proc. IEEE Int. Conf. Neural Netw., 1. Orlando, FL, Jun.-Jul. 1994, pp. $55-60$.

[38] M. Opper, O. Winther. A Bayesian approach to online learning. On-line Learning in Neural Networks, ed. D. Saad (1998), 363-378.

[39] I. Ortigosa, R. Lopez and J. Garcia. A neural networks approach to residuary resistance of sailing yachts prediction. In Proceedings of the International Conference on Marine Engineering MARINE 2007, 2007.

[40] C. Scheffler. A derivation of the EM updates for finding the maximum likelihood parameter estimates of the Student's $t$ distribution. Working Paper, http://www.inference.org.uk/cs482/publications/scheffler2008derivation.pdf (2008).

[41] J. Soch, C. Allefeld. Kullback-Leibler divergence for the normal-gamma distribution. arXiv:1611.01437 [math.ST] (2016).

[42] D. M. Titterington. Bayesian methods for neural netowrks and related models. Statistical Science, 19, No. 1 (2004), 128-139.

[43] P. Tüfekci, Prediction of full load electrical power output of a base load operated combined cycle power plant using machine learning methods, International Journal of Electrical Power and Energy Systems, 60 (2014), 126-140.

[44] G. Uludag, A. S. Uyar, K. Senel, H. Dag, Comparison of evolutionary techniques for value-at-risk calculation. In: Giacobini M. (eds) Applications of Evolutionary Computing. EvoWorkshops 2007. Lecture Notes in Computer Science, vol 4448. Springer, Berlin, Heidelberg. 2007.

[45] R. D. De Veaux, J. Schumi, J. Schweinsberg, L. H. Ungar, Prediction intervals for neural networks via nonlinear regression, Technometrics, 40, no. 4, (1998), 273-282.

[46] M. Welling, Y. W. Teh, Bayesian learning via stochastic gradient Langevin dynamics, In Proceedings of the 28th International Conference on Machine Learning, 28 June - 2 July 2011, Bellevue, Washington, USA, 681 (2011)

[47] C. J. Wild and G. A. F. Seber, Nonlinear Regression. New York, Wiley, 1989.

[48] I-C. Yeh. Modeling of strength of high performance concrete using artificial neural networks, Cement and Concrete Research, 28, No. 12 (1998), 1797-1808. 